\title{
Agrocybe aegerita serves as a gateway for identifying sesquiterpene biosynthetic enzymes in higher fungi
}

Congqiang Zhang, ${ }^{1,}$, Xixian Chen ${ }^{1}$, Axel Orban ${ }^{2}$, Sudha Shukal ${ }^{1}$, Florian Birk ${ }^{2}$, Heng-Phon Too ${ }^{1,3}$, Martin Rühl2,4,

${ }^{1}$ Biotransformation Innovation Platform (BioTrans), Agency for Science, Technology and Research (A*STAR), Singapore; ${ }^{2}$ Institute of Food Chemistry and Food Biotechnology, Justus Liebig University Giessen, Giessen, Germany; ${ }^{3 D}$ epartment of Biochemistry, Yong Loo Lin School of Medicine, National University of Singapore, Singapore; 4Fraunhofer Institute for Molecular Biology and Applied Ecology, Branch for Bioresources, Giessen Germany

*To whom correspondence should be addressed.

Congqiang Zhang: BioTrans, A*STAR; zcqsimon@outlook.com, congqiang zhang@biotrans.a-star.edu.sg; Martin Rühl: Institute of Food Chemistry and Food Biotechnology, Justus Liebig University Giessen, 35392 Giessen, Germany; martin.ruehl@uni-giessen.de;

SUPPORTING INFORMATION: 


\section{SUPPLEMENTARY METHODS}

Analysis of volatile terpenoids during fructification. Agrocybe aegerita wildtype-strain AAE-3 was grown at $24{ }^{\circ} \mathrm{C}$ in the dark in modified crystallizing dishes as illustrated (1) (Figure 1; lower dish: $70 \mathrm{~mm}$ in diameter, upper dish: $80 \mathrm{~mm}$ in diameter; glass pipe attached to the upper dish: outer diameter $16 \mathrm{~mm}$, inner diameter $14 \mathrm{~mm}$ ) with $16 \mathrm{~mL} 1.5 \% \mathrm{MEA}$ (containing $15 \mathrm{~g}$ malt extract and $15 \mathrm{~g}$ agar per liter) and sealed with Parafilm ${ }^{\mathrm{TM}}$. In ten days after inoculation, the mycelium covered the complete agar surface. The Parafilm ${ }^{\mathrm{TM}}$ was removed and the samples were transferred to a climate chamber $\left(24{ }^{\circ} \mathrm{C}, 95 \% \mathrm{rH}, 12 / 12 \mathrm{~h}\right.$ day/night rhythm) and cultured for another 16 days. Volatile organic compounds were collected by solid phase microextraction (SPME) using a divinylbenzene-carboxen-polydimethylsiloxane $(50 / 30 \quad \mu \mathrm{m}$ DVB/CAR/PDMS) fiber (length $1 \mathrm{~cm}$; Supelco). Beginning with day 10 after inoculation, volatiles were absorbed directly in the crystallizing dishes for $14 \mathrm{~h}$ (7/7 h day/night). This extraction was carried out every second day. For GC-MS analysis, an Agilent Technologies 7890A gas chromatograph equipped with a VF-WAXms column (Agilent Technologies; $30 \mathrm{~m} x$ $0.25 \mathrm{~mm}, 0.25 \mu \mathrm{m}$ ) and connected to an Agilent 5975C MSD Triple-Axis mass spectrometer (MS) was used. Helium was used as gas carrier, with a flow rate of $1.2 \mathrm{~mL} \mathrm{x} \mathrm{min}^{-1}$. Mass spectra were acquired in the mass range of 33-300 $\mathrm{m} / \mathrm{z}$. Ionization was performed by electron impact at $70 \mathrm{eV}$ with an ion source temperature set at $230{ }^{\circ} \mathrm{C}$. The SPME fiber was inserted into the injector of the gas chromatograph for thermal desorption in splitless mode for 1 min, with the injector temperature held at $250^{\circ} \mathrm{C}$. The GC oven temperature was programmed to ramp from $40^{\circ} \mathrm{C}$ (held for 3 min) to $240{ }^{\circ} \mathrm{C}$ (held for $7 \mathrm{~min}$ ) at $5{ }^{\circ} \mathrm{C} \times \min ^{-1}$.

Structural elucidation of $\Delta^{6}$-protoilludene. Transformed E. coli Agr7 was cultured in LB media with 34 mg L ${ }^{-1}$ chloramphenicol, $50 \mathrm{mg} \mathrm{L}^{-1}$ kanamycin and $50 \mathrm{mg} \mathrm{L}^{-1}$ spectinomycin overnight at $37^{\circ} \mathrm{C}$ and $180 \mathrm{rpm}$. Of this culture, $20 \mathrm{~mL}$ were inoculated in $800 \mathrm{~mL}$ culture medium $\left(15 \mathrm{~g} \mathrm{~L}^{-1}\right.$ glucose, $2 \mathrm{~g} \mathrm{~L}^{-1}\left(\mathrm{NH}_{4}\right)_{2} \mathrm{SO}_{4}, 4.2 \mathrm{~g} \mathrm{~L}^{-1} \mathrm{KH}_{2} \mathrm{PO}_{4}$, $11.24 \mathrm{~g} \mathrm{~L}^{-1} \mathrm{~K}_{2} \mathrm{HPO}_{4}$, $1.7 \mathrm{~g} \mathrm{~L}^{-1}$ citric acid, $0.5 \mathrm{~g} \mathrm{~L}^{-1} \mathrm{MgSO}_{4}$, $10 \mathrm{~mL}$ trace element solution: $0.25 \mathrm{~g} \mathrm{~L}^{-1} \mathrm{CoCl}_{2} \cdot 6 \mathrm{H}_{2} \mathrm{O}, 1.5 \mathrm{~g} \mathrm{~L}^{-1} \mathrm{MnSO}_{4} \cdot 4 \mathrm{H}_{2} \mathrm{O}$, o.15 g L-1

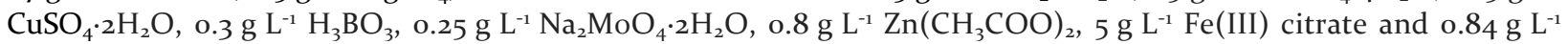
EDTA). Cultivation was performed for 3 days in a $1 \mathrm{~L}$ gas washing bottle at $24{ }^{\circ} \mathrm{C}$ with aeration set at $3 \mathrm{~L} \mathrm{~h}^{-1}$ and agitation at $250 \mathrm{rpm}$. As $\mathrm{OD}_{6 \text { oo }}$ reached 0.6 , the culture was induced with o.1 $\mathrm{mM}$ IPTG and the outgoing air was led through a gas washing bottle filled with hexane to trap produced $\Delta^{6}$-protoilludene. The hexane was dried with $\mathrm{Na}_{2} \mathrm{SO}_{4}$ and was removed using a rotary evaporator. After determination of yield ( $34 \mathrm{mg}$ ) and purity (>95\%) by means GC-MS, $\Delta^{6}$-protoilludene was dissolved in $\mathrm{CDCl}_{3}$ and the structure was elucidated by nuclear magnetic resonance (NMR). The NMR experiments $\left({ }^{1} \mathrm{H},{ }^{13} \mathrm{C},{ }^{1} \mathrm{H}\right.$ COSY, HSQC, HMBC) were performed on a Bruker Avance III 6oo MHz. The data of the chemical shift are in accordance with the literature $(2,3)$, whereby the assignment of the corresponding carbon atoms in the literature is not correct. The assignment of two methyl groups (12 and 14) is incorrect. In this study, the respective assignment of the methyl groups were corroborated by an additional NOESY experiment. 
SUPPLEMENTARY FIGURES
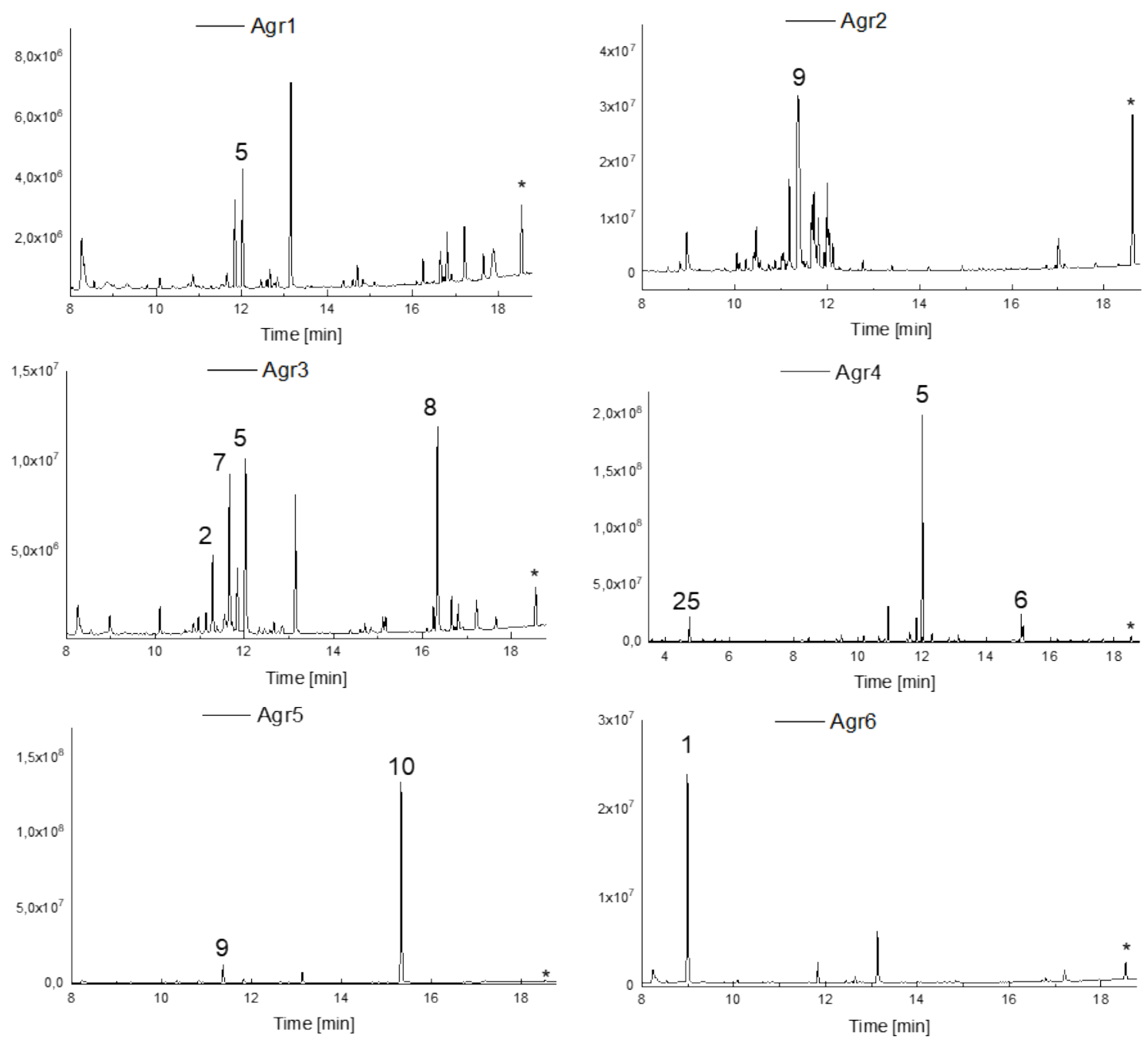

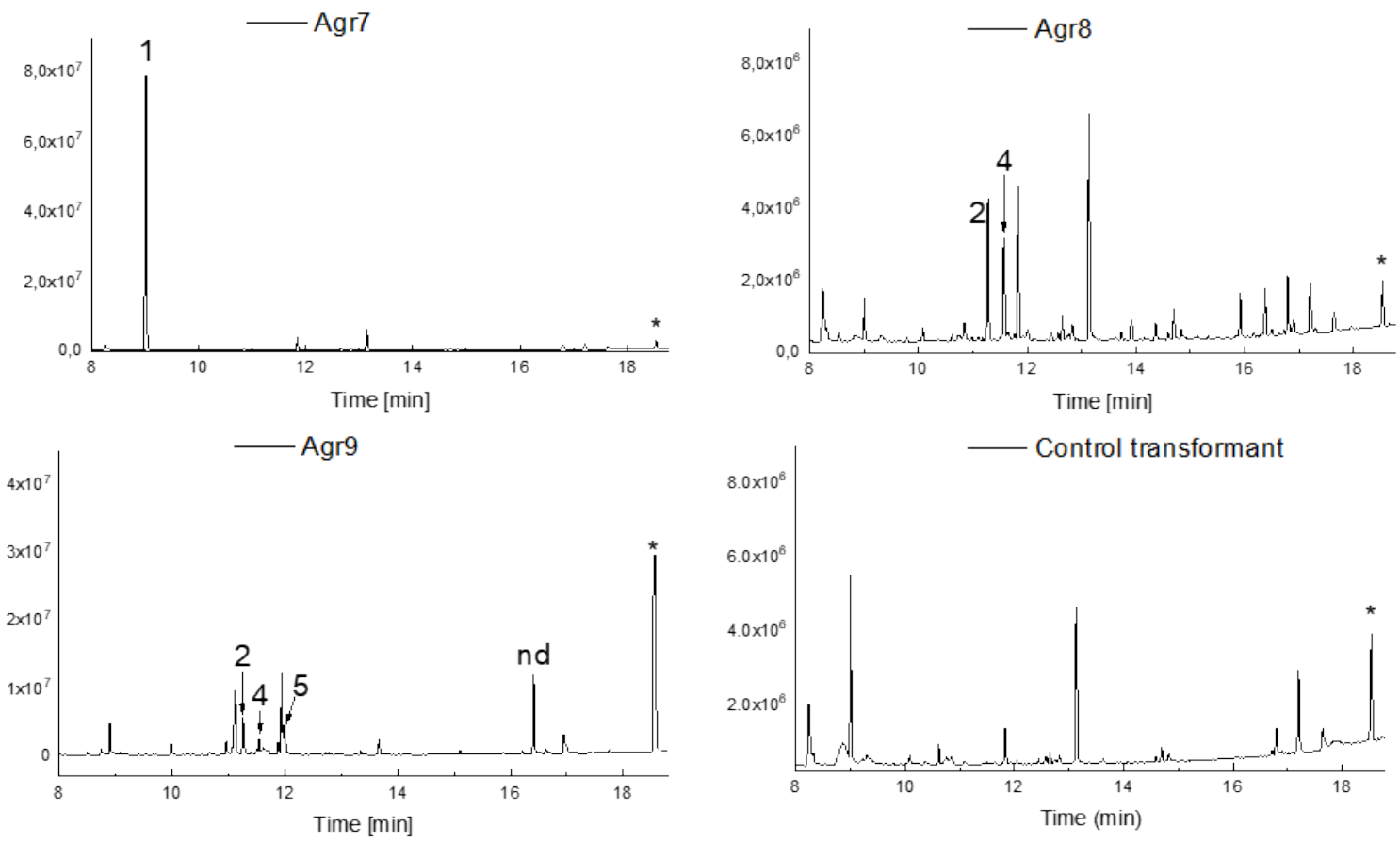

Supplementary Figure 1. Terpenes produced by E. coli expressing TPS genes from A. aegerita. The metabolites were analyzed by means of GC-MS using a VF-WAXms column. Control transformant is the GC-MS profile of volatile metabolites produced by an E. coli clone carrying an empty vector. Indole $\left(^{*}\right)$ endogenously produced by $E$. coli serves as an internal reference. 

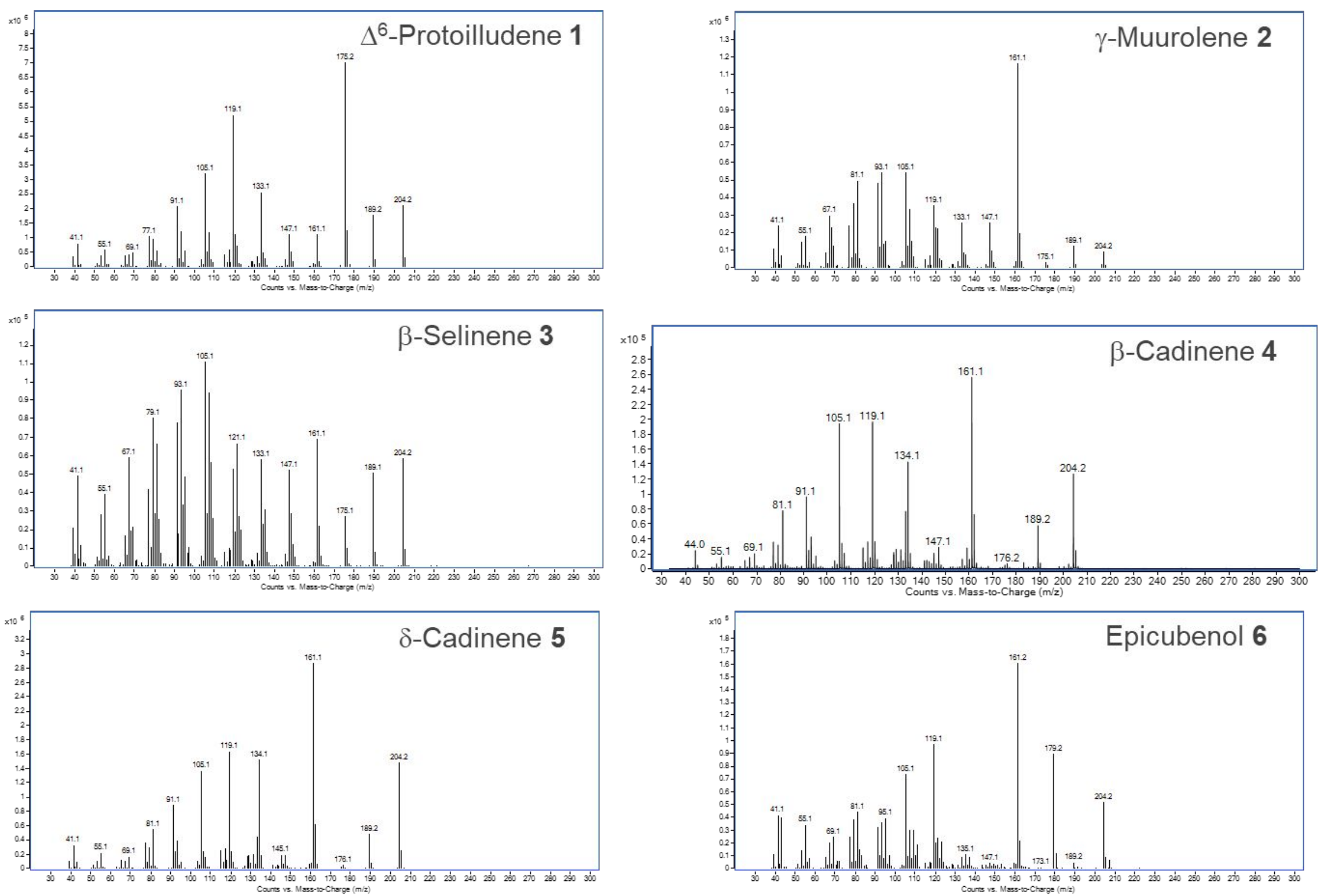

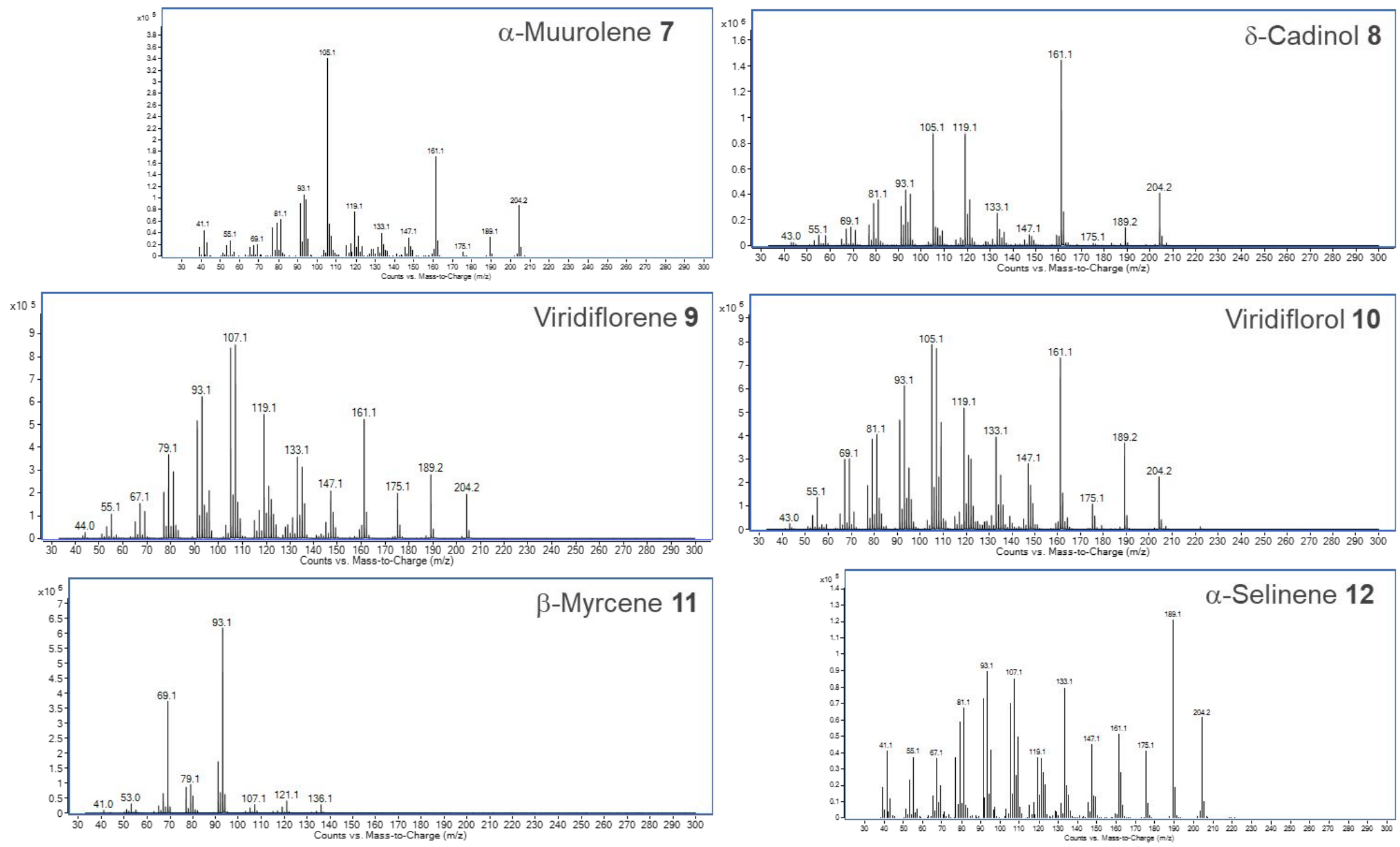

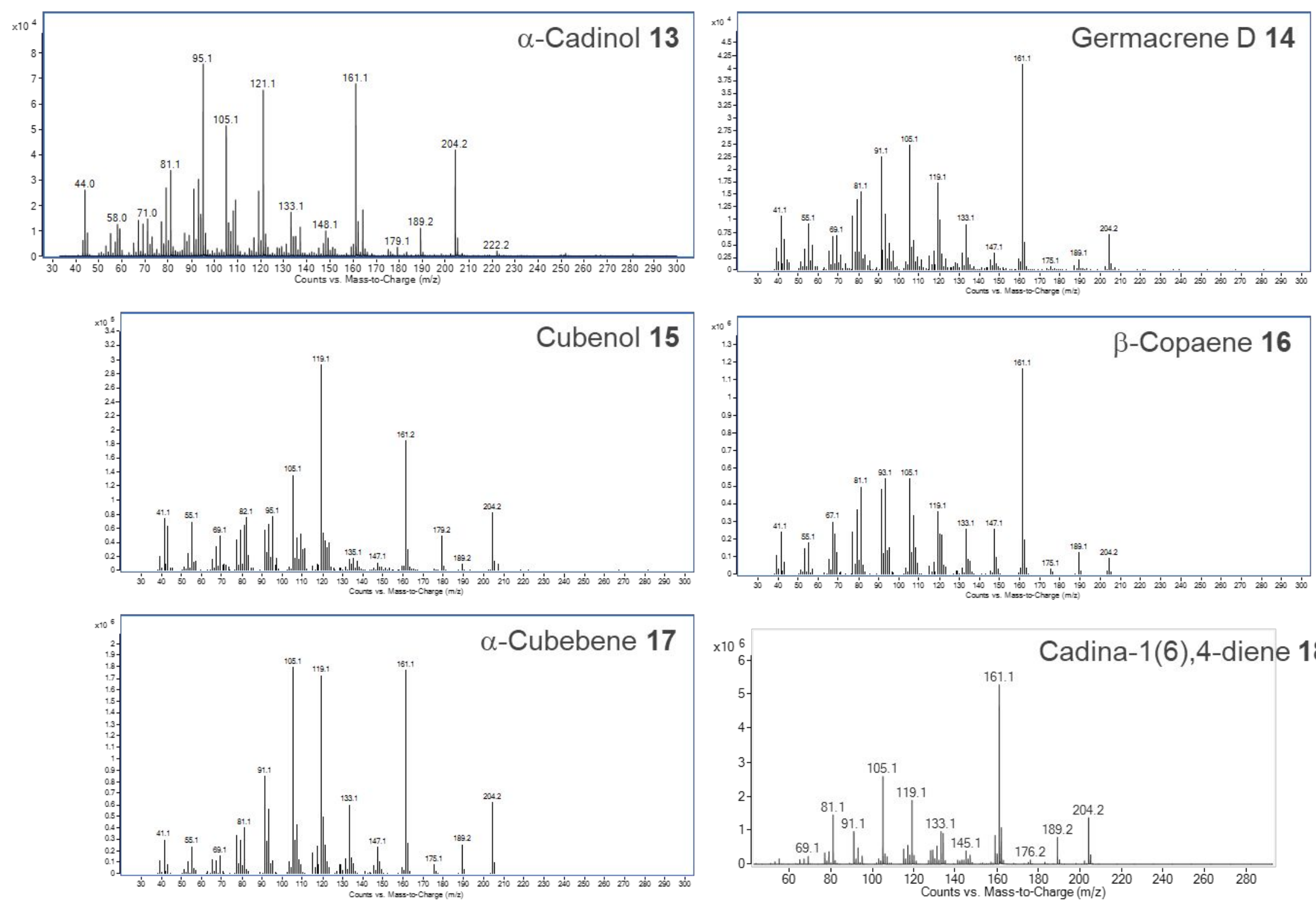

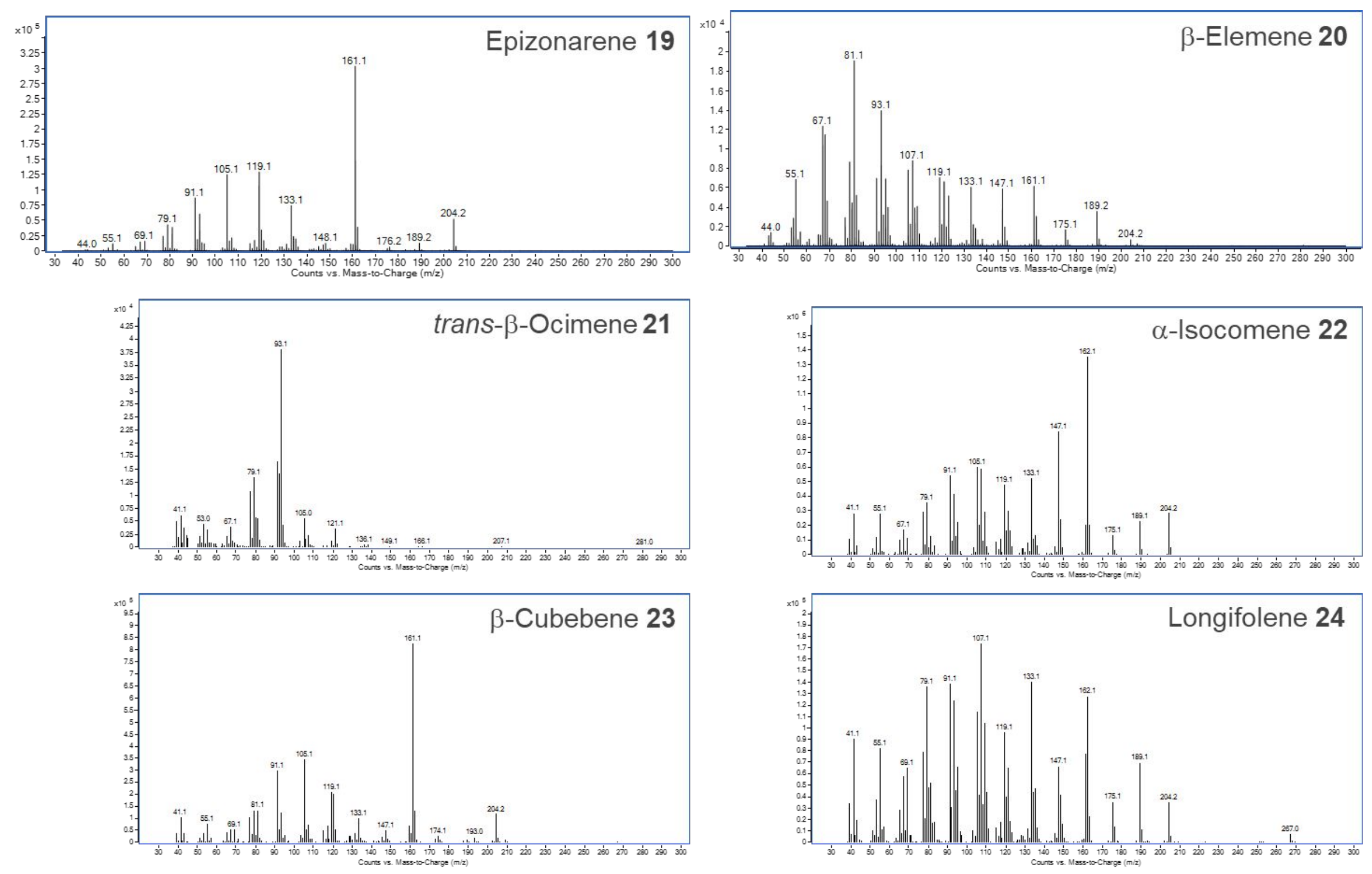

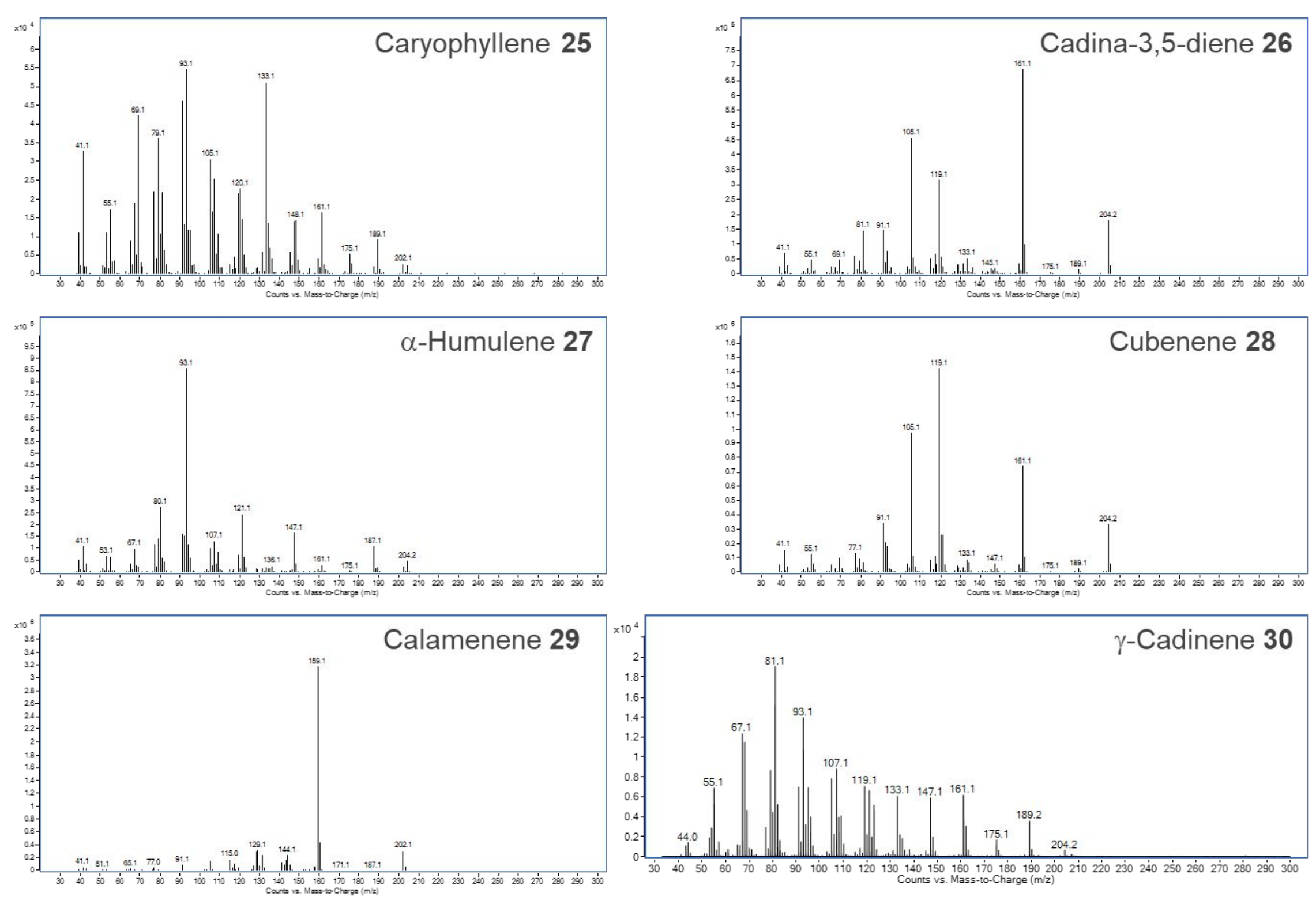

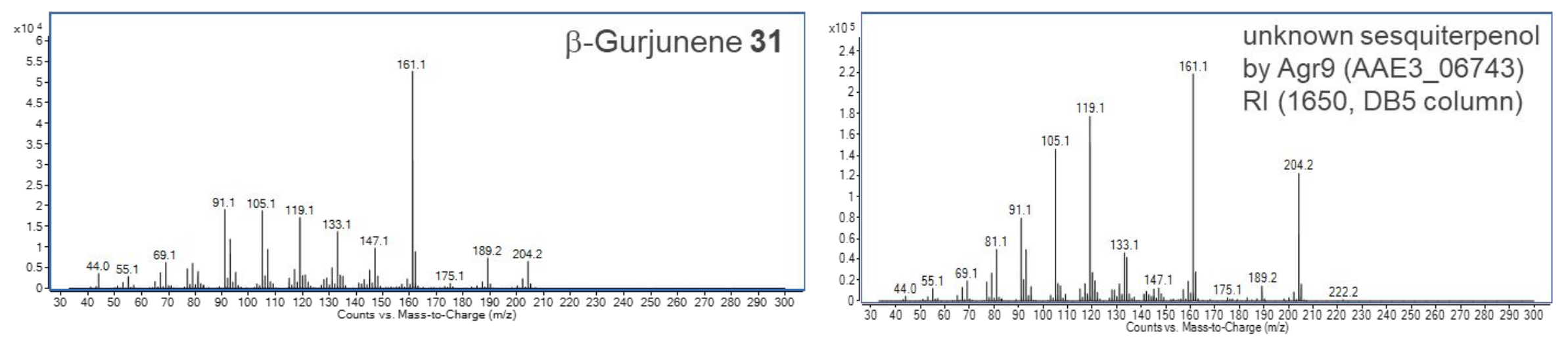

Supplementary Figure 2. Mass spectra of terpene compounds analyzed in this study. 


\begin{tabular}{|c|c|}
\hline 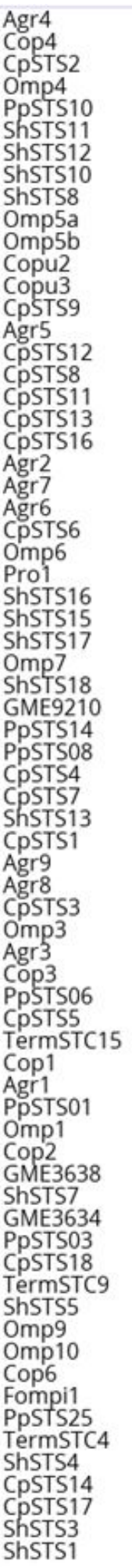 & 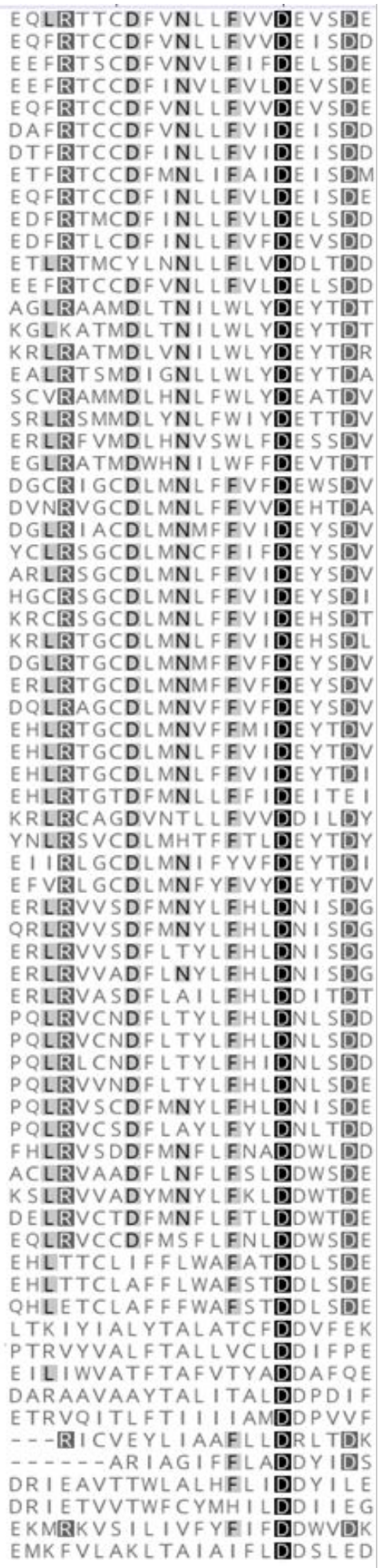 \\
\hline $\begin{array}{l}\text { Agr10 } \\
\text { Agr11 }\end{array}$ & 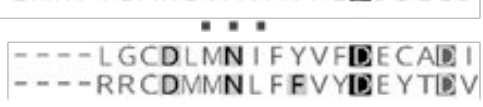 \\
\hline
\end{tabular}

A YWAACDH V CWANDV YSYYD MEQS KEL A YWAACDF VCWANDV YISYDMEQ K KGH I YWAA VDL VCWTINDV YSYYDMEQ S KE I

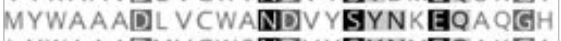
L YWAAADMV CWS NDIV Y SYYNMIEQAKIG I MYWA S ADIMV CWANDV YSY NVEQAKGH VYWAA I DIMVWC ANDIV YSYY S MEL A KIGD I YWAA VDIMVWWANDV YSYK VEQAK GL L NL AA VDIMV CWANDI YSY DMEQAK GL MYWA S VDL VCWS NDV YSIYNVEQA K IGH MYWAS VDL VCW SINDV YSYN VEQ A KIGH VYAD S ADIMV I L VINDV FISYNRIEQAK GL VYFCAADMVCWS NDVYSYYNMEQ AKIGH GYNAAMDL VFWANDL FISYNMEQAKIGH GYNAGMDL VFWAIND L VSYNMEQ S KIGH GYNAAMDL VFWVNDL YSYNMEQ E KEH GYNATMDL ACWVINDVYISYNMEQ EAKIGH AYDAALDLAFGTNDI HSYNMEQSKDH AYNAALDLAFGTNDI OSYNMIEQAKOH AYNGALDL GY I VNDI HSYNMEQSK[GH GY I AALDL I FL ANDL Y YSYNMEQAKIGH L TAYC I DML C I GND L CSYNVIES R GD LREWA I DIML I L GND]L CSYNVIAQSR RD L LEL CVDM I L I GNDL YSYNVIGQARED L S LWC I DML I I GNDL C CSYNVEQAHGD LRK SC I DML CL GNDIV VSYN L GQARDD LSLAT I DML CL GND] I VSYNLLEARGD L RMAT I DMML CL GND I VSYN I [AQAR [GD LHVLS I SM I CL GND I VSWN L IAQ SRED L A I L A I DM I L L GNDT ASYYNYIAQ ARIGD LSAL STDM I CL GNDI CSYNVIGQARIED LTTWA I DM I I L GND] I VIYNVIEQARIGD LSYN I AEL I I LDND I ASYNKKEQATED LSLYATEMLI I DNDL VISYNREQASEATVTA I DML | | GNDL CSFRNAHARIGD AVT S L TDML I I ANDV FSF K VIA SRDD L I A I I QD I SC I ANDT F SIYRRIGQSRIGR LARDTTDM I VL ANDIMCEYKKIA I ADD LREQSTDL I A I GND] DSYAM[KAR[GL. LTEAAVDL I I L VNDMHSYVREL SCGH LNQSTNDL VTWS NDI FEYNVEQSRIGD LNOS TNDL VTWSNDI FSYNVYAOSRED LNQ GTNDL VTWS NDI F SYYNVIEQSRIGD

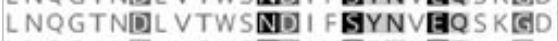
LNOSANDL VSWSNDI YSFNNEQAH[GL GEAANDL VTWS NDI FISYNVIGQS K [GD LGEAANDL VTWS NDI F SYYNVIQ S KED L GEAANDL VTWS ND] I FSF NVAQ S K [GD L GEATNDL VTWS ND] I FEY S V局Q S K [GH LGEAANDL VTWS ND I F ISYNVIGQS KEGD METAANDVVSFAND I YSFN I GQS K [GD LEDASNDL I AWS ND I FSFNREQSRHD LEREANAC I SWS NDLL FISYNVEQARIGD LQDATNDL VTWS NDI FISYNVIEQSRIGD MADATNDL Y SWANDV LSYNAIEQ SRIGD L LDATNDCV SWANDI LSYNREQSRIGD MSQAT SDIMTWPIDDL CSF NKIEQADIGD MSOVT SDI MTWPNDL CISF NKIEQADIGD MSEATTDI MTWPNDL CSFNKIEQADIGD - LPDMR I Y I NHVNDVL ISF YKIEDKAIGE - FPDLYTYANYVINDVLISFYK目D I AGE - FPEQIDY I NGTINDL LISF YKI目E LDCE VFPDICFFLNHTNDI LSFYK目TLDIGE - I PDAMLYVSYVINDI LISFYKI国ELAIGE MEEQF I DL I SLAND L YSYYKKGY I AKD AE G I VSDHVGL TNDF FISY L K目KMTNS FFKLVSDHLVL VNDI YSYK VEQDRSE L LRVSGRHI AVANDL FISYR V目ALRAG AQVLGSEAGVLRNEASISYVKGVNE[GS SMPDM I YY I ELVINDL MS F YKIEQLAGE $\because *$

ALREQAPFL- - -[D] NSSYPMK - VR[GL LEE I YTR -......... TRV S SICQ

Supplementary Figure 3. Alignment of amino acid sequences of characterized terpene synthases and the two nonfunctional STS Agrio and Agrı. Please see Figure 2 for abbreviations. Accession numbers of amino acid sequences can be taken from Supplementary Table 2 and 3. 


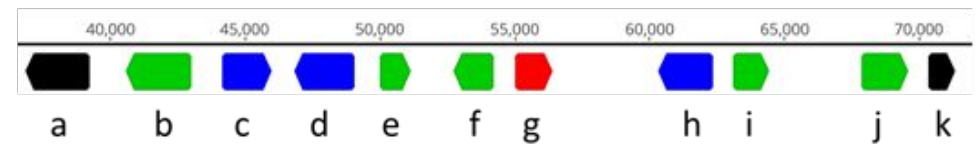

ID Top hits against NCBI Reference Proteins database

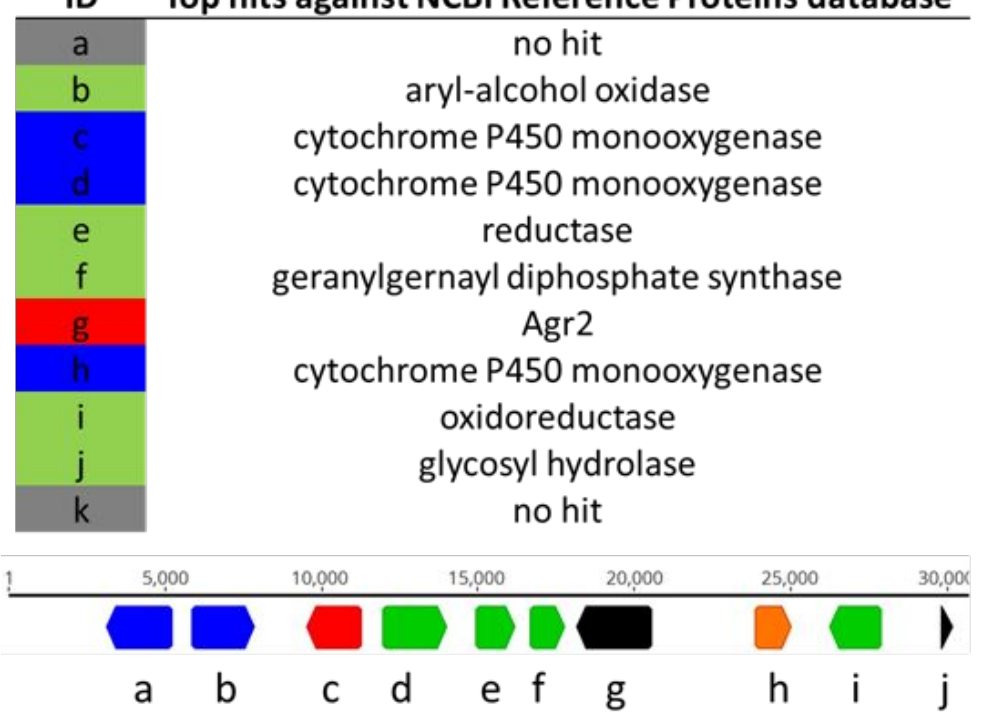

ID Top hits against NCBI Reference Proteins database

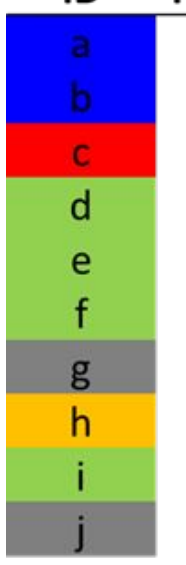

cytochrome P450 monooxygenase

cytochrome P450 monooxygenase

$$
\text { Agr6 }
$$

putative diels-alderase

aldo-keto reductase

kinase-like protein

hypothetical protein

membrane fraction protein

alpha/beta-hydrolase

no hit

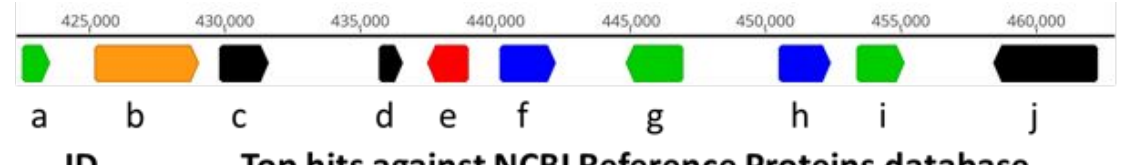

ID Top hits against NCBI Reference Proteins database

\begin{tabular}{cc}
\hline a & hydrolase \\
b & vacuolar membrane protein \\
c & hypothetical protein \\
d & no hit \\
e & Agr5 \\
\hline f & cytochrome P450 monooxygenase \\
\hline g & oxidoreductase \\
\hline h & cytochrome P450 monooxygenase \\
\hline i & oxidoreductase \\
\hline$j$ & transcription elongation factor
\end{tabular}

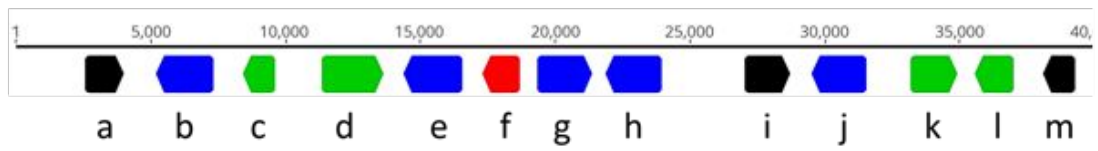

ID Top hits against NCBI Reference Proteins database

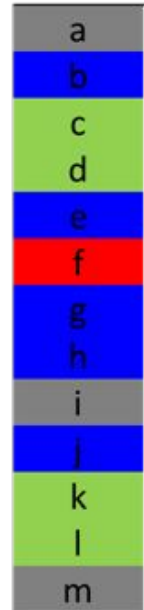

hypothetical protein

cytochrome P450 monooxygenase

aldo-keto reductase

GMC oxidoreductase

cytochrome P450 monooxygenase

$$
\text { Agr7 }
$$

cytochrome P450 monooxygenase

cytochrome P450 monooxygenase

no hit

cytochrome P450 monooxygenase

aldo-keto reductase

$S$-adenosyl-L-methionine-dependent methyltransferase

$\mathrm{NAD}(\mathrm{P})$-bindung protein

Supplementary Figure 4. Predicted biosynthetic TPS gene clusters in A. aegerita. 

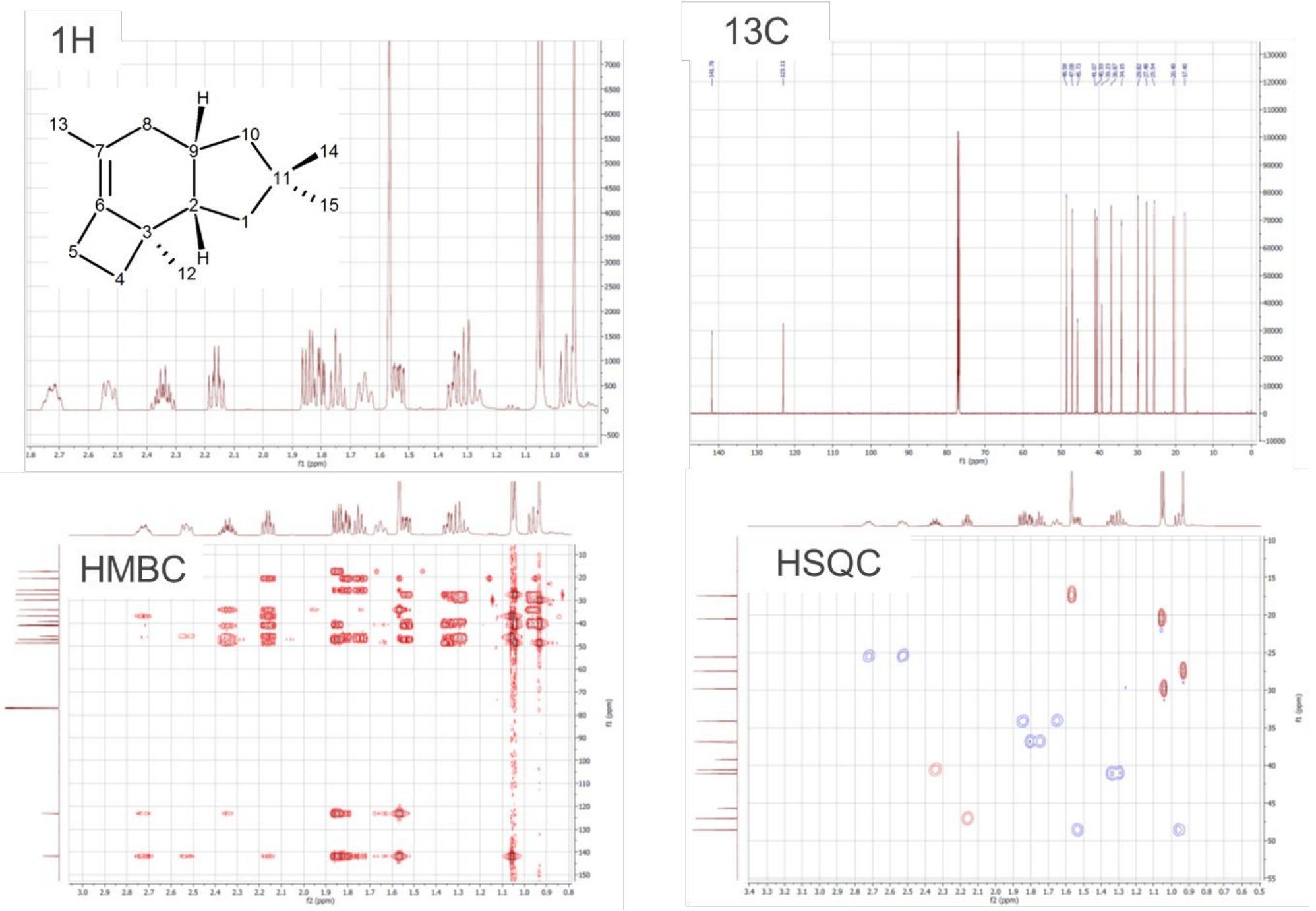

Supplementary Figure 5. NMR analysis of purified $\Delta^{6}$-protoilludene as described in the Supplementary Method section 


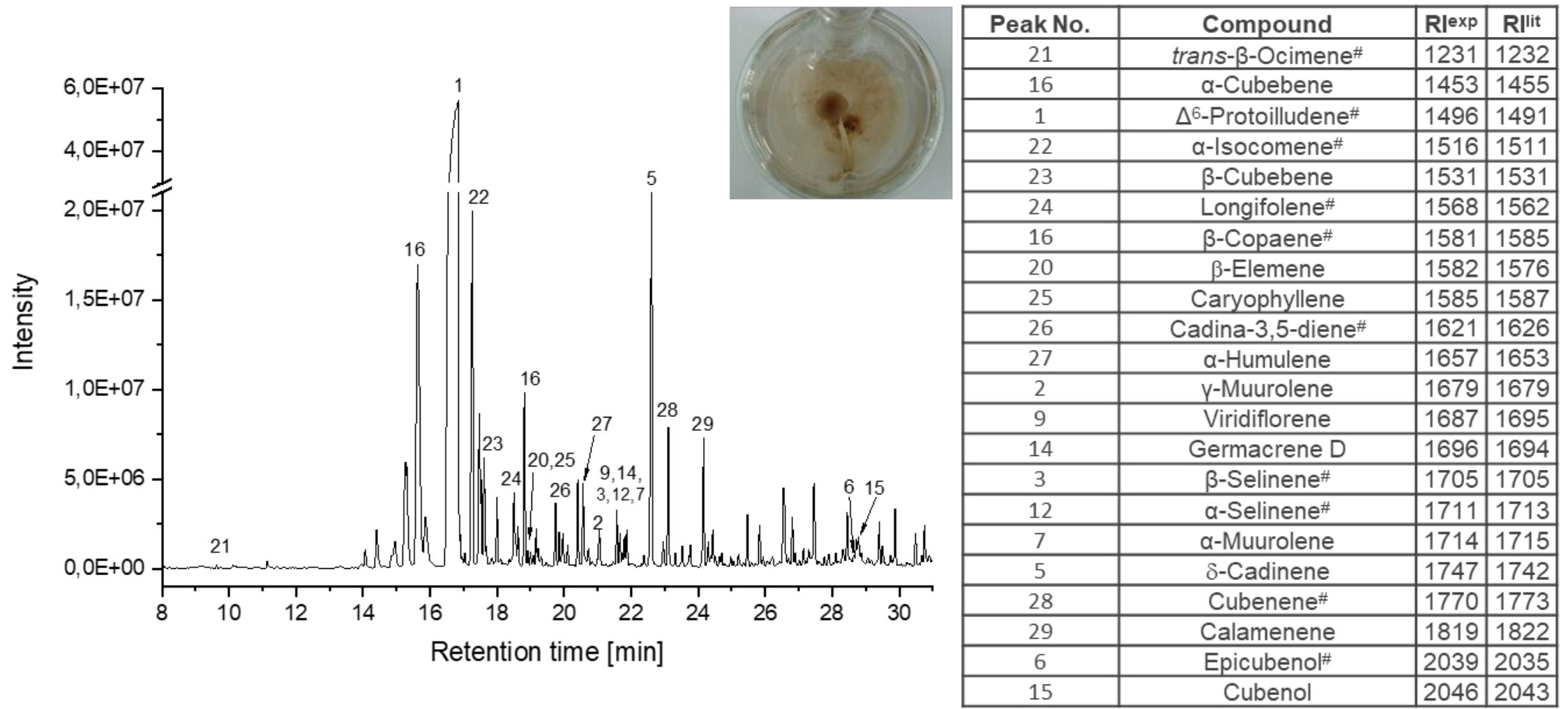

Supplementary Figure 6. Volatile terpenoids produced by A. aegerita during sporulation.Volatile metabolites from A. aegretia culture were sampled by solid phase microextraction (SPME) and analyzed by means of GC-MS (VF-WAXms) as descreibed in Supporting Methods. Compounds tentatively identified on basis of their retention indices (RIs) and mass spectra in comparison to those in the literature and databases are marked with a hashmark (\#). All other compounds are verified by additional comparison with authentic standards or essential oils. The mass spectra of detected terpene compounds and their structures are shown in Supplementary Figures 2 and 7 , respectively. 


(1)

Supplementary Figure 7. Terpene compounds detected in this study. Compounds are tentatively identified (black number) or identified by comparing with an authentic standard and/or essential oils (green numbers). 


\section{VF-WAXms}
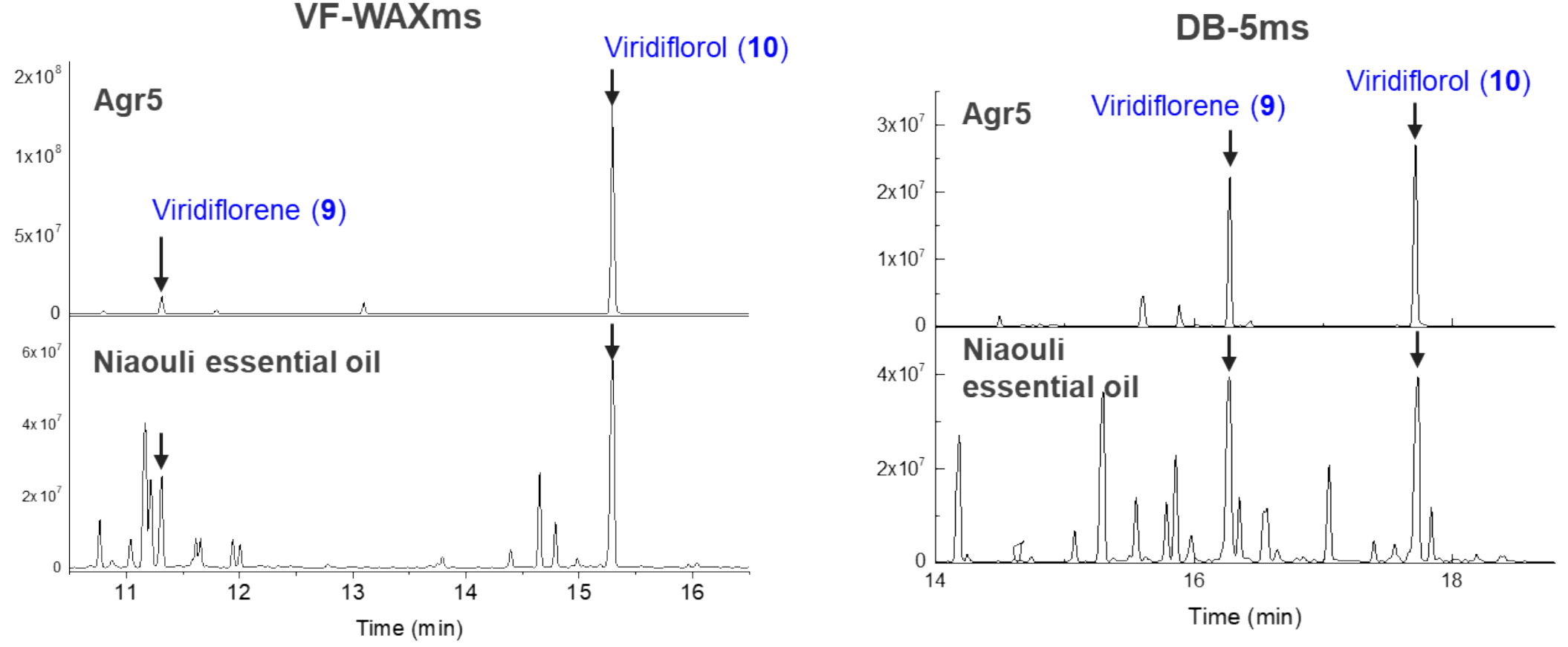

Supplementary Figure 8. Chromatograms of GC-MS analysis on a VF-WAXms and a DB5-ms column of Niaouli essential oil used as chemical standard to identify viridiflorene and viridiflorol in E. coli clones expressing A. aegerita STSs Agr5. 


\section{$\alpha$-Muurolene (7) $\delta$-Cadinene (5)}
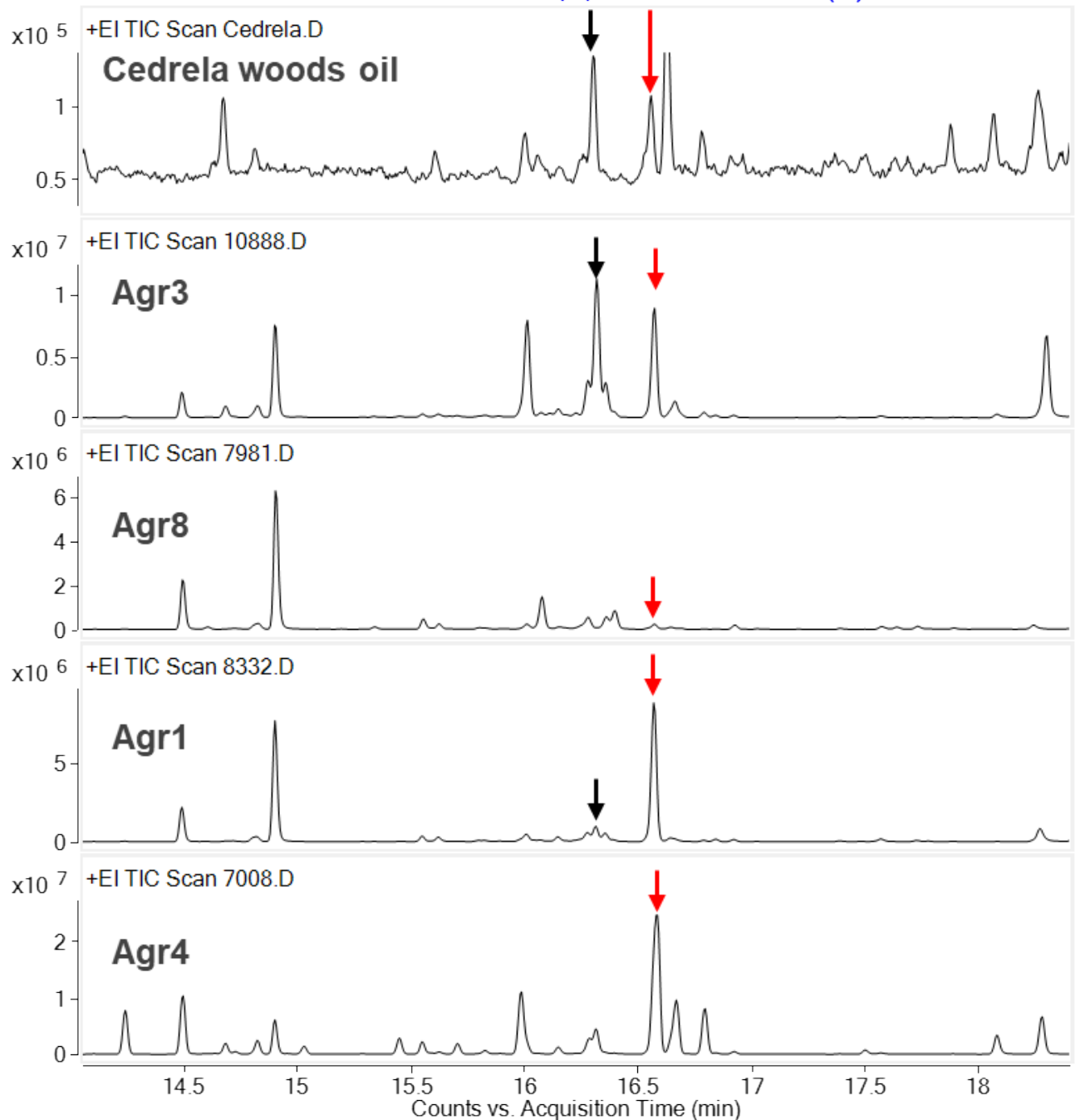

Supplementary Figure 9. Chromatograms of GC-MS analysis on a DB5-ms column of Cedrela woods oil used as chemical standard to identify sesquiterpenes 16 and 17 in $E$. coli clones expressing A. aegerita STSs Agr1, Agr3, Agr4 and Agr8. 


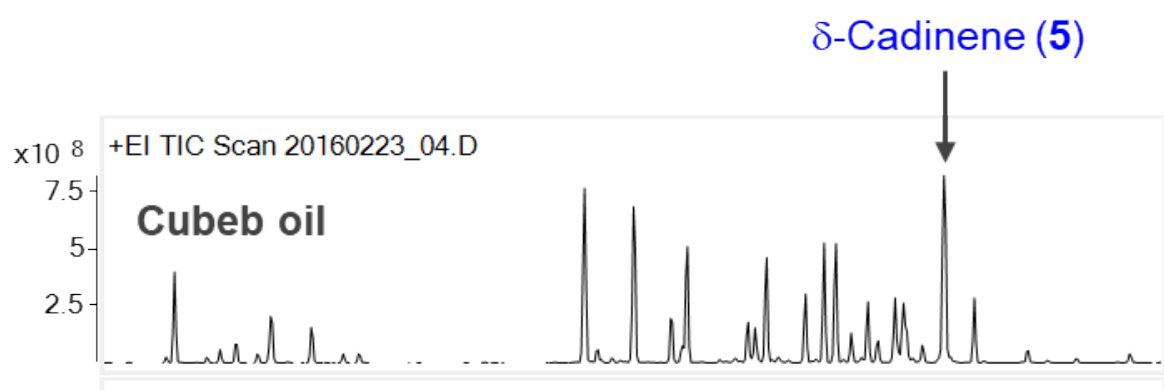

x10 7 +EI TIC Scan 10888.D

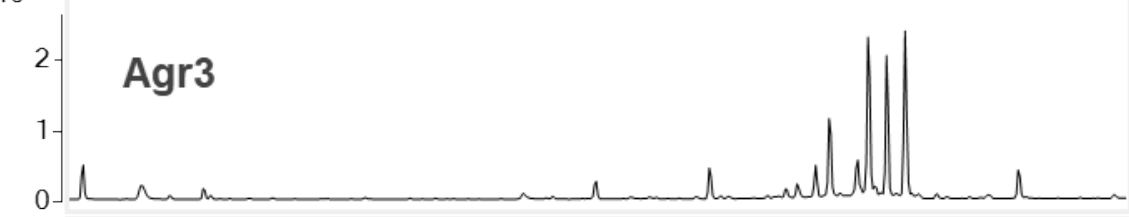

x10 8 +EI TIC Scan 7008.D

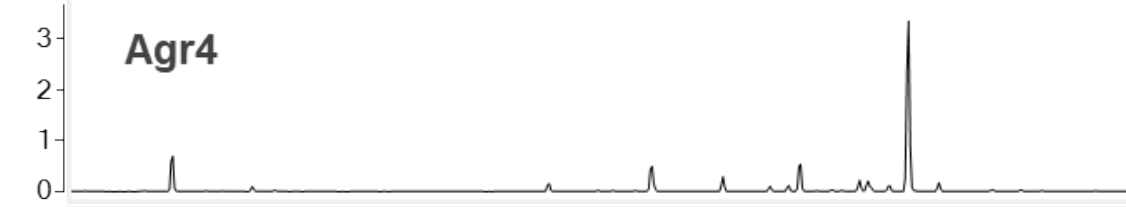

x10 6 +EI TIC Scan 8332.D

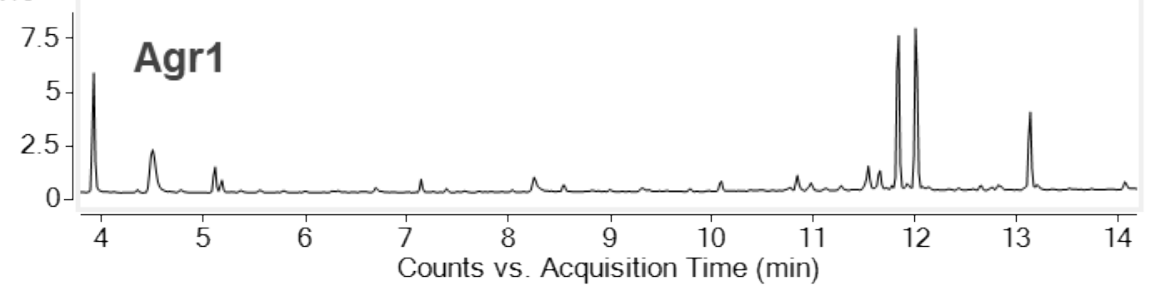

Germacrene D (14)

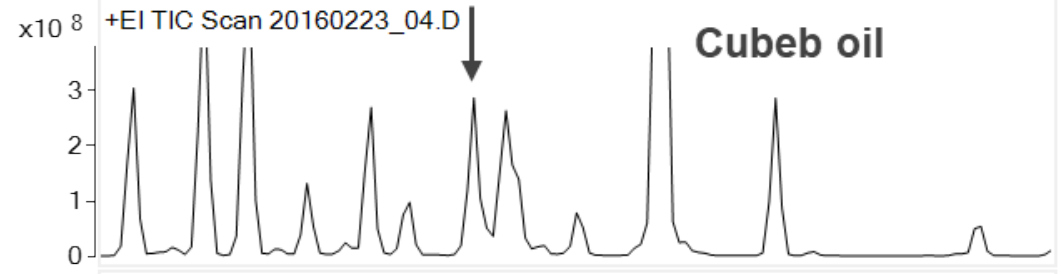

x10 7 +EI TIC Scan 10888.D

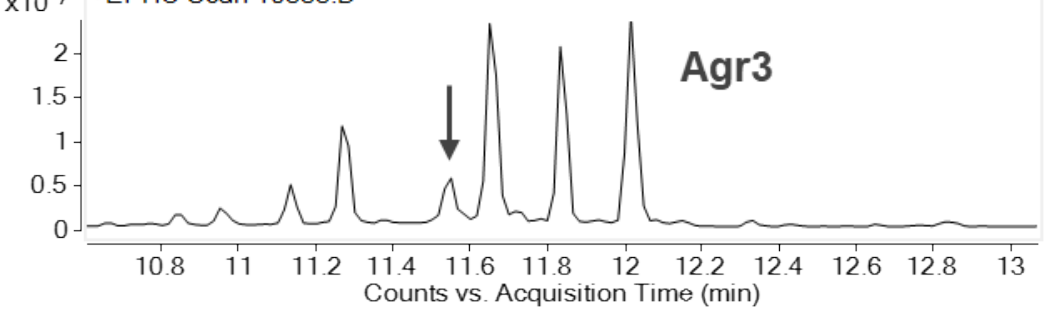

Supplementary Figure 1o. Chromatograms of GC-MS analysis on a VF-WAXms column of Cubeb oil used as chemical standard to identify sesquiterpenes $\mathbf{1 3}$ and $\mathbf{1 7}$ in E. coli clones expressing A. aegerita STSs Agrı, Agr3 and Agr4. 

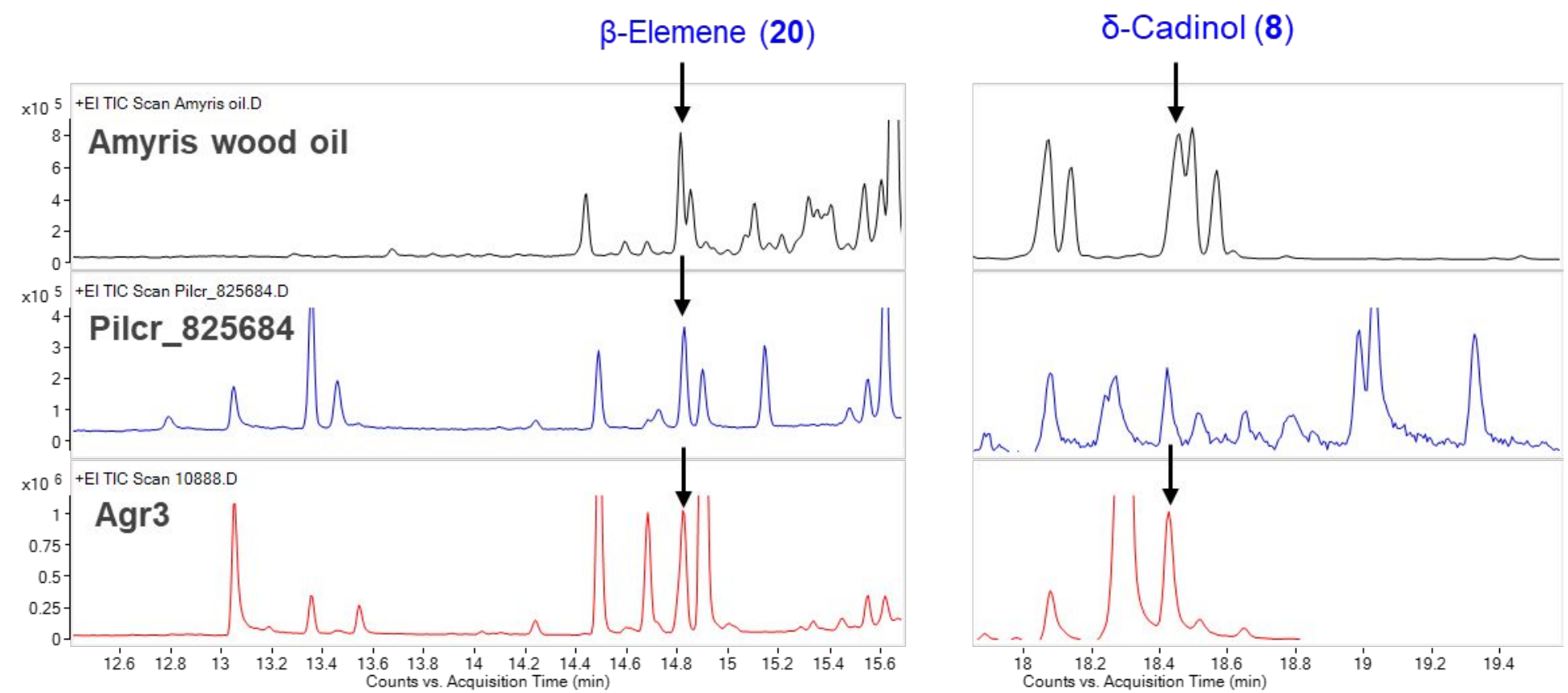

Supplementary Figure 11. Chromatograms of GC-MS analysis on a VF-WAXms and a DB-5ms column of Amyris wood oil used as chemical standard to identify $\beta$ elemene and $\delta$-cadinol in E. coli clones expressing A. aegerita STS Agr3 and Piloderma croceum STS Pilcr_825684. 


\section{Supplementary Tables}

Supplementary Table 1. Genes coding for TPS in Agrocybe aegerita.

\begin{tabular}{|c|c|c|c|c|c|c|c|c|}
\hline TPS & $I D^{*}$ & $\begin{array}{l}\text { Accession } \\
\text { number }\end{array}$ & Scaffold & Gene start & Gene stop & Gene length & $\begin{array}{ll}\begin{array}{l}\text { Number } \\
\text { introns }\end{array} & \text { of } \\
\end{array}$ & $\begin{array}{l}\text { Protein } \\
\text { length }\end{array}$ \\
\hline Agr1 & 06595 & MN146024 & 28 & 329,611 & 328,403 & 1,209 & 3 & 346 \\
\hline Agr2 & 12839 & MN146025 & 70 & 55,035 & 56,437 & 1,403 & 4 & 389 \\
\hline Agr3 & 13190 & MN146026 & 8 & 106,456 & 107,896 & 1,441 & 6 & 358 \\
\hline Agr4 & 09164 & MN146027 & 4 & 405,253 & 406,500 & 1,248 & 4 & 342 \\
\hline Agr5 & 13291 & MN146028 & 8 & 439,057 & 437,487 & 1,571 & 5 & 430 \\
\hline Agr6 & 04120 & MN146029 & 2 & 11,372 & 10,043 & 1,330 & 5 & 346 \\
\hline Agr7 & 10454 & MN146030 & 49 & 18,741 & 17,315 & 1,427 & 5 & 387 \\
\hline Agr8 & 04444 & MN146031 & 2 & $1,035,120$ & $1,033,830$ & 1,291 & 4 & 353 \\
\hline Agr9 & 06743 & MN146032 & 29 & 231,813 & 233,188 & 1,376 & 4 & 372 \\
\hline Agr10 & 09008 & MN146033 & 39 & 349,082 & 347,841 & 1,242 & 6 & 308 \\
\hline Agr11 & 05024 & MN146034 & 21 & 112,812 & 111,488 & 1,325 & 4 & 355 \\
\hline
\end{tabular}

* ID refers to the annotated TPS gene (AAE3_ID) in the A. aegerita genome (https://www.thineslab.senckenberg.de/agrocybe genome).

Supplementary Table 2. Products of recombinant TPSs analyzed in this study

\begin{tabular}{|c|c|c|c|c|c|c|c|}
\hline \multirow{2}{*}{$\begin{array}{l}\text { TPS } \\
\text { (Gene ID) }\end{array}$} & \multirow{2}{*}{ Products } & \multicolumn{3}{|c|}{ DB5 (non-polar) } & \multicolumn{3}{|c|}{ VF-WAXms (polar) } \\
\hline & & Area \% & RI & Literature RI & Area \% & RI & Literature RI \\
\hline \multirow{7}{*}{$\begin{array}{l}\text { Agr1 } \\
\text { (AAE3_06595) }\end{array}$} & $\gamma$-Muurolene 2 & $5 \%$ & 1489 & $1477 \pm 3(392)$ & I & 1 & $1692 \pm 12(165)$ \\
\hline & $\beta$-Selinene 3 & $5 \%$ & 1508 & $1486 \pm 3(349)$ & 1 & 1 & $1717 \pm 13(167)$ \\
\hline & $\alpha$-Muurolene 7 & $7 \%$ & 1511 & $1499 \pm 3(427)$ & $8 \%$ & 1739 & $1726 \pm 13(198)$ \\
\hline & $\alpha$-Selinene 12 & $5 \%$ & 1514 & 1517 & 1 & 1 & $1656 \pm 0(2)$ \\
\hline & $\delta$-Cadinene 5 & $60 \%$ & 1529 & $1524 \pm 2(751)$ & $57 \%$ & 1772 & $1758 \pm 13(374)$ \\
\hline & $\delta$-Cadinol 8 & $8 \%$ & 1659 & $1635 \pm 15$ & $13 \%$ & 2198 & $2187 \pm 10$ \\
\hline & $\alpha$-Cadinol 13 & $10 \%$ & 1671 & $1653 \pm 2(400)$ & $18 \%$ & 2243 & $2226 \pm 9(182)$ \\
\hline $\begin{array}{l}\text { Agr2 } \\
\text { (AAE3_12839) }\end{array}$ & Viridiflorene 9 & $82 \%$ & 1508 & $1493 \pm 4(114)$ & $81 \%$ & 1712 & $1697 \pm 7(76)$ \\
\hline \multirow{6}{*}{$\begin{array}{l}\text { Agr3 } \\
\text { (AAE3_13190) }\end{array}$} & $\gamma$-Muurolene 2 & $22 \%$ & 1489 & $1477 \pm 3(392)$ & $9 \%$ & 1706 & $1692 \pm 12(165)$ \\
\hline & Germacrene D 14 & 1 & 1 & 1 & $4 \%$ & 1730 & $1710 \pm 14(325)$ \\
\hline & $\alpha$-Muurolene 7 & $32 \%$ & 1512 & $1499 \pm 3(427)$ & $20 \%$ & 1740 & $1726 \pm 13(198)$ \\
\hline & $\delta$-Cadinene 5 & $24 \%$ & 1529 & $1524 \pm 2(751)$ & $24 \%$ & 1772 & $1758 \pm 13(374)$ \\
\hline & Cubenol 15 & 1 & 1 & 1 & $4 \%$ & 2076 & $2080 \pm 4(65)$ \\
\hline & $\delta$-Cadinol 8 & $21 \%$ & 1662 & $1635 \pm 15$ & $24 \%$ & 2209 & $2187 \pm 10$ \\
\hline \multirow{9}{*}{$\begin{array}{l}\text { Agr4 } \\
\text { (AAE3_09164) }\end{array}$} & $\beta$-Myrcene 11 & $10 \%$ & 989 & $991 \pm 2(841)$ & $7 \%$ & 1172 & $1161 \pm 7(569)$ \\
\hline & $\beta$-Copaene 16 & 1 & 1 & 1 & $2 \%$ & 1610 & $1586 \pm 12(15)$ \\
\hline & $\alpha$-Cubebene 17 & $8 \%$ & 1358 & $1351 \pm 2(338)$ & $1 \%$ & 1469 & $1463 \pm 6(186)$ \\
\hline & Cadina-1(6),4-diene 18 & 1 & 1 & 1 & $8 \%$ & 1677 & $1692 \pm 12(165)$ \\
\hline & $\gamma$-Muurolene 2 & $15 \%$ & 1487 & $1477 \pm 3(392)$ & 1 & 1 & 1 \\
\hline & $\delta$-Cadinene 5 & $37 \%$ & 1531 & $1524 \pm 2(751)$ & $59 \%$ & 1772 & $1758 \pm 13(374)$ \\
\hline & Epizonarene 19 & $13 \%$ & 1538 & $1501 \pm 4(28)$ & 1 & 1 & 1 \\
\hline & Epicubenol 6 & $8 \%$ & 1660 & $1627 \pm 2(144)$ & $7 \%$ & 2076 & $2067 \pm 21(67)$ \\
\hline & Cubenol 15 & 1 & 1 & 1 & $4 \%$ & 2081 & 2080 \\
\hline \multirow{2}{*}{$\begin{array}{l}\text { Agr5 } \\
\text { (AAE3_13291) }\end{array}$} & Viridiflorene 9 & $42 \%$ & 1509 & $1493 \pm 4(114)$ & $9 \%$ & 1714 & $1697 \pm 7(76)$ \\
\hline & Viridiflorol 10 & $58 \%$ & 1617 & $1591 \pm 2(198)$ & $91 \%$ & 2099 & $2095 \pm 10(108)$ \\
\hline $\begin{array}{l}\text { Agr6 } \\
\text { (AAE3_04120) }\end{array}$ & $\Delta^{6}$-Protoilludene 1 & $100 \%$ & 1391 & 1393 & $100 \%$ & 1513 & 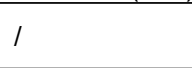 \\
\hline $\begin{array}{l}\text { Agr7 } \\
\text { (AAE3 10454) }\end{array}$ & $\Delta^{6}$-Protoilludene 1 & $100 \%$ & 1392 & l & $100 \%$ & 1513 & l \\
\hline
\end{tabular}




\begin{tabular}{|c|c|c|c|c|c|c|c|}
\hline \multirow{2}{*}{$\begin{array}{l}\text { TPS } \\
\text { (Gene ID) }\end{array}$} & \multirow{2}{*}{ Products } & \multicolumn{3}{|c|}{ DB5 (non-polar) } & \multicolumn{3}{|c|}{ VF-WAXms (polar) } \\
\hline & & Area \% & RI & Literature RI & Area \% & RI & Literature RI \\
\hline \multirow{7}{*}{$\begin{array}{l}\text { Agr8 } \\
\text { (AAE3_04444) }\end{array}$} & $\beta$-Elemene 20 & $8 \%$ & 1400 & $1391 \pm 2(521)$ & 1 & 1 & $1591 \pm 9(259)$ \\
\hline & $\gamma$-Muurolene 2 & $33 \%$ & 1487 & $1477 \pm 3(392)$ & $30 \%$ & 1706 & $1692 \pm 12(165)$ \\
\hline & $\beta$-Selinene 3 & $18 \%$ & 1509 & $1486 \pm 3(349)$ & 1 & l & $1717 \pm 13(167)$ \\
\hline & $\alpha$-Selinene 12 & $14 \%$ & 1515 & 1517 & 1 & 1 & $1656 \pm 0(2)$ \\
\hline & $\beta$-Cadinene 4 & $21 \%$ & 1518 & $1518 \pm 10(30)$ & $22 \%$ & 1733 & $1720 \pm N / A(1)$ \\
\hline & $\delta$-Cadinene 5 & $6 \%$ & 1529 & $1524 \pm 2(751)$ & $4 \%$ & 1772 & $1758 \pm 13(374)$ \\
\hline & $\alpha$-Cadinol 13 & I & 1 & 1 & $9 \%$ & 2213 & $2226 \pm 9(182)$ \\
\hline \multirow{4}{*}{$\begin{array}{l}\text { Agr9 } \\
\text { (AAE3_06743) }\end{array}$} & $\gamma$-Muurolene 2 & $27 \%$ & 1488 & $1477 \pm 3(392)$ & $14 \%$ & 1706 & $1692 \pm 12(165)$ \\
\hline & $\beta$-Cadinene 4 & $13 \%$ & 1516 & $1518 \pm 10(30)$ & $13 \%$ & 1733 & $1720 \pm N / A(1)$ \\
\hline & $\delta$-Cadinene 5 & $13 \%$ & 1527 & $1524 \pm 2(751)$ & $6 \%$ & 1772 & $1758 \pm 13(374)$ \\
\hline & unknown sesquiterpenol & $44 \%$ & 1650 & 1 & $52 \%$ & 2176 & \\
\hline (Denbi1_659367) & $\Delta^{6}$-Protoilludene 1 & $100 \%$ & 1391 & 1393 & $100 \%$ & 1513 & 1 \\
\hline (Hetan2_454193) & $\Delta^{6}$-Protoilludene 1 & $100 \%$ & 1391 & 1393 & $100 \%$ & 1513 & 1 \\
\hline (Hypsu1_138665*) & $\Delta^{6}$-Protoilludene 1 & $100 \%$ & 1392 & 1393 & $100 \%$ & 1514 & 1 \\
\hline \multirow{2}{*}{ (Denbi1_816208) } & Viridiflorene 9 & $31 \%$ & 1509 & $1493 \pm 4(114)$ & $8 \%$ & 1714 & $1697 \pm 7(76)$ \\
\hline & Viridiflorol 10 & $57 \%$ & 1617 & $1591 \pm 2(198)$ & $92 \%$ & 2099 & $2095 \pm 10(108)$ \\
\hline \multirow{2}{*}{ (Sphst_47084) } & Viridiflorene 9 & $32 \%$ & 1509 & $1493 \pm 4(114)$ & $8 \%$ & 1714 & $1697 \pm 7(76)$ \\
\hline & Viridiflorol 10 & $58 \%$ & 1617 & $1591 \pm 2(198)$ & $92 \%$ & 2099 & $2095 \pm 10(108)$ \\
\hline \multirow{3}{*}{ (Pilcr_825684) } & $\beta$-Elemene 20 & $7 \%$ & 1401 & $1391 \pm 2(521)$ & 1 & 1 & $1591 \pm 9(259)$ \\
\hline & Viridiflorene 12 & $6 \%$ & 1509 & $1493 \pm 4(114)$ & $8 \%$ & 1714 & $1697 \pm 7(76)$ \\
\hline & $\gamma$-Cadinene 30 & $45 \%$ & 1529 & $1513 \pm 2(485)$ & $30 \%$ & 1774 & $1765 \pm 11(199)$ \\
\hline (Galma_104215) & $\beta$-Gurjunene 31 & $83 \%$ & 1441 & $1432 \pm 3(234)$ & $81 \%$ & 1673 & $1669 \pm 17(14)$ \\
\hline
\end{tabular}

Mass spectra of all products are available in Supplementary Figure S2. Numbers in brackets in the columns refer to the protein IDs in the respective genome browser $(4,5)$. Literature RI data are from NIST/EPA/NIH Mass Spectral Library, version: NIST vi7.

\section{Supplementary Table 3. Published and functionally characterized fungal terpene synthases}

\begin{tabular}{|c|c|c|c|c|}
\hline $\begin{array}{l}\text { Accession No or } \\
\text { JGI Protein ID }\end{array}$ & Terpene synthase & Organism & Main product(s) & Reference \\
\hline \multicolumn{5}{|l|}{ BASIDIOMYCOTA } \\
\hline MN146024 & Agr1 & \multirow{9}{*}{ Agrocybe aegerita } & $\delta$-Cadinene & \multirow{9}{*}{ this study } \\
\hline MN146025 & Agr2 & & Viridiflorene & \\
\hline MN146026 & Agr3 & & $\alpha$-Muurolene & \\
\hline MN146027 & Agr4 & & $\delta$-Cadinene & \\
\hline MN146028 & Agr5 & & Viridiflorol & \\
\hline MN146029 & Agr6 & & $\Delta^{6}$-Protoilludene & \\
\hline MN146030 & Agr7 & & $\Delta^{6}$-Protoilludene & \\
\hline MN146031 & Agr8 & & $\gamma$-Muurolene & \\
\hline MN146032 & Agr9 & & unknown sesquiterpenol & \\
\hline AGR34199 & Pro1 & Armillaria gallica & $\Delta^{6}$-Protoilludene & (6) \\
\hline LC436345 & CpSTS1 & \multirow{13}{*}{$\begin{array}{l}\text { Clitopilus } \\
\text { pseudo-pinsitus }\end{array}$} & Sterpurene & \multirow{13}{*}{ (7) } \\
\hline LC436346 & CpSTS2 & & $\delta$-Cadinene & \\
\hline LC436347 & CpSTS3 & & $\alpha$-Muurolene, $\delta$-Cadinol & \\
\hline LC436348 & CpSTS4 & & $\Delta^{6}$-Protoilludene & \\
\hline LC436349 & CpSTS5 & & $\gamma$-Muurolene, $\alpha$-Muurolene & \\
\hline LC436350 & CpSTS6 & & Pentalenene & \\
\hline LC436351 & CpSTS7 & & $\alpha$-Farnesene & \\
\hline LC436352* & CpSTS8 & & Alloaromadendrene & \\
\hline LC436353 & CpSTS9 & & Ledene, Viridiflorol & \\
\hline LC436355 & CpSTS11 & & 9-Alloaromadendrene & \\
\hline LC436356 & CpSTS12 & & Ledene, Viridiflorol & \\
\hline LC436357 & CpSTS13 & & Ledene, Viridiflorol & \\
\hline LC436358 & CpSTS14 & & $\beta$-elemene, $\beta$-farnesene & \\
\hline
\end{tabular}




\begin{tabular}{|c|c|c|c|c|}
\hline LC436360 & CpSTS16 & & Aristolene & \\
\hline LC436361 & CpSTS17 & & $\beta$-Caryophyllene & \\
\hline LC436362 & CpSTS18 & & $\gamma$-Cadinene & \\
\hline XP_007771895 & Copu2 & \multirow{2}{*}{ Coniophora puteana } & $\beta$-Copaene & \multirow{2}{*}{ (8) } \\
\hline XP_007765978 & Copu3 & & Cubebol & \\
\hline EAU89322 & Cop1 & \multirow{5}{*}{ Coprinopsis cinerea } & Germacrene A & \multirow{5}{*}{ (9) } \\
\hline EAU85264 & Cop2 & & Germacrene A & \\
\hline EAU88892 & Cop3 & & $\alpha$-Muurolene & \\
\hline EAU85540 & Cop4 & & $\delta$-Cadinene & \\
\hline EAU89298 & Cop6 & & $\alpha$-Cuprenene & \\
\hline JGI ID: Denbi1_659367 & Denbi1_659367 & \multirow{2}{*}{ Dendrothele bispora } & $\Delta^{6}$-Protoilludene & \multirow{2}{*}{ this study } \\
\hline JGI ID: Denbi1_816208 & Denbi1_816208 & & Viridiflorol & \\
\hline JGI ID: 84944 & Fompi1 & Fomitopsis pinicola & $\alpha$-Cuprenene & (10) \\
\hline JGI ID: Galma_104215 & Galma_104215 & Galerina marginata & $\beta$-Gurjunene & \multirow{3}{*}{ this study } \\
\hline JGI ID: Hetan2_454193 & Hetan2_454193 & Heterobasidion annosum & $\Delta^{6}$-Protoilludene & \\
\hline JGI ID: Hypsu1_138665 & Hypsu1_138665 & Hypholoma sublateritium & $\Delta^{6}$-Protoilludene & \\
\hline KX281943 & GME3634 & \multirow{3}{*}{ Lignosus rhinocerotis } & $\alpha$-Cadinol & \multirow{3}{*}{ (11) } \\
\hline KX281944 & GME3638 & & Torreyol & \\
\hline KX281945 & GME9210 & & $\begin{array}{l}\text { 1,3,4,5,6,7-Hexahydro-2,5,5- } \\
\text { trimethyl-2H-2,4a-ethanonaphthalene }\end{array}$ & \\
\hline JGI ID: 1311 & Omp1 & \multirow{8}{*}{ Omphalotus olearius } & $\alpha$-Muurolene & \multirow{8}{*}{ (10) } \\
\hline JGI ID: 4636 & Omp3 & & $\alpha$-Muurolene & \\
\hline JGI ID: 1447 & Omp4 & & $\delta$-Cadinene & \\
\hline JGI ID: $2392 / 3$ & Omp5a/b & & $\gamma$-Cadinene & \\
\hline JGI ID: 4774 & Omp6 & & $\Delta^{6}$-Protoilludene & \\
\hline JGI ID: 2271 & Omp7 & & $\Delta^{6}$-Protoilludene & \\
\hline JGI ID: 3258 & Omp9 & & $\alpha$-Barbatene & \\
\hline JGI ID: 3981 & Omp10 & & (E)-Dauca-4(11),8-diene & \\
\hline JGI ID: Pilcr_825684 & Pilcr_825684 & Piloderma croceum & $\gamma$-Cadinene & this study \\
\hline JGI ID: Pospl1_60326 & PpSTS01 & \multirow{7}{*}{ Postia placenta } & $\alpha$-Muurolene, $\delta$-Cadinene, $\beta$-Elemene & \multirow{7}{*}{$(12)$} \\
\hline JGI ID: Pospl1_99496 & PpSTS03 & & $\alpha$-Cadinene, $\gamma$-Cadinene & \\
\hline JGI ID: Pospl1_45581 & PpSTS06 & & $\alpha$-Gurjunene & \\
\hline JGI ID: Pospl1_59374 & PpSTS08 & & $\Delta^{6}$-Protoilludene & \\
\hline JGI ID: Pospl1_98072 & PpSTS10 & & $\delta$-Cadinene & \\
\hline JGI ID: Pospl1_101549* & PpSTS14 & & Pentalenene & \\
\hline JGI ID: Pospl1_125961 & PpSTS25 & & linalool, myrcene & \\
\hline JGI ID: Sphst_47084 & Sphst_47084 & Sphaerobolus stellatus & Viridiflorol & this study \\
\hline JGI ID: 159379 & ShSTS1 & \multirow{13}{*}{ Stereum hirsutum } & $\alpha$-Barbatene, $\beta$-Barbatene & \multirow{13}{*}{$(7,13)$} \\
\hline JGI ID: 122776 & ShSTS3 & & $\beta$-Farnesebe, $\alpha$-farnesene & \\
\hline JGI ID: 52743 & ShSTS4 & & Hirsutene & \\
\hline JGI ID: 161672 & ShSTS5 & & $\gamma$-Cadinene & \\
\hline JGI ID: 167646 & ShSTS7 & & $\delta$-Cadinene & \\
\hline JGI ID: 146390 & ShSTS8 & & $\alpha$-Cubebene, 1-epi-Cubenol & \\
\hline JGI ID: 111121 & ShSTS10 & & Germacrene D, $\delta$-Cadinene & \\
\hline JGI ID: 128017 & ShSTS11 & & $\delta$-Cadinene & \\
\hline JGI ID: 111127 & ShSTS12 & & $\alpha$-Cubebene, $\beta$-Cubebene & \\
\hline JGI ID: 50042 & ShSTS13 & & $\beta$-Caryophyllene & \\
\hline JGI ID: 64702 & ShSTS15 & & $\Delta^{6}$-Protoilludene & \\
\hline JGI ID: 73029 & ShSTS16 & & $\Delta^{6}$-Protoilludene & \\
\hline JGI ID: 69906 & ShSTS17 & & $\Delta^{6}$-Protoilludene & \\
\hline
\end{tabular}




\begin{tabular}{|c|c|c|c|c|}
\hline JGI ID: 25180 & ShSTS18 & & $\Delta^{6}$-Protoilludene & \\
\hline not available & STC4 & \multirow{3}{*}{ Termitomyces } & Intermedeol & \multirow{3}{*}{ (14) } \\
\hline KNZ74377 & STC9 & & $\gamma$-Cadinene & \\
\hline not available & STC15 & & Germacrene D-4-ol & \\
\hline \multicolumn{5}{|l|}{ ASCOMYCOTA } \\
\hline not available & FlvE & Aspergillus flavus & $(+)$-Acoradiene & (15) \\
\hline AAF13264 & AtAS & Aspergillus terreus & Aristolochene & (16) \\
\hline AAQ16575 & BcBOT2 & \multirow{2}{*}{ Botrytis cinerea } & Presilphiperfolan-8b-ol & (17) \\
\hline XP_024550392 & BcABA3 & & $\alpha$-lonylideneethane & (18) \\
\hline JGI ID:17536 & EC12-PGS & \multirow{4}{*}{ Daldinia eschscholzii EC12 } & Guaiene, Pinene & \multirow{4}{*}{$(19)$} \\
\hline JGI ID:315006 & EC12-GS & & Gurnunene & \\
\hline JGI ID:24646 & EC12-SS & & Selinene & \\
\hline JGI ID:70183 & EC12-ILS & & IsoLedene & \\
\hline not provided & Dia1 & Diaporthe sp. & $\Delta^{6}$-Protoilludene & (20) \\
\hline ССT65043 & FfSTC3 & \multirow{2}{*}{ Fusarium fujikuroi } & (+)-Eremophilene & \multirow{2}{*}{$(21)$} \\
\hline ССT75704 & FfSTC5 & & (-)-Guaia-6,10(14)-diene & \\
\hline CCP20071 & Ffsc6 & \multirow{2}{*}{ Fusarium fujikuroi } & $(-)$ - $\alpha$-Acorenol & \multirow{2}{*}{$(22)$} \\
\hline CCP20072 & Ffsc4 & & Koraiol & \\
\hline ACY69978 & FgCLM1 & Fusarium graminearum & Longiborneol & (23) \\
\hline AAD13657 & FsTDS & Fusarium sporotrichioides & Trichodiene & (24) \\
\hline AHY23920 & Hyp1 & \multirow{3}{*}{ Hypoxylon sp. } & (E)-Nerolidol & \multirow{3}{*}{$(25)$} \\
\hline AHY23921 & Hyp2 & & $\delta$-Cadinene & \\
\hline AHY23922 & Hyp3 & & 1,8-Cineole & \\
\hline JGI ID:6706 & $\mathrm{Cl} 4 \mathrm{~A}-\mathrm{CS}$ & \multirow{2}{*}{ Hypoxylon sp. Cl4A } & Caryophyllene & \multirow{6}{*}{ (19) } \\
\hline JGI ID:322581 & Cl4A-CPS & & Chamigrene, Pinene & \\
\hline JGI ID:397991 & CO27-CS & \multirow{2}{*}{ Hypoxylon sp. CO27 } & Caryophyllene & \\
\hline JGI ID:392541 & CO27-CPS & & Chamigrene, Pinene & \\
\hline JGI ID:372695\# & EC38-CS & \multirow{2}{*}{ Hypoxylon sp. EC38 } & Caryophyllene & \\
\hline JGI ID:424147\# & EC38-CPS & & Chamigrene, Pinene & \\
\hline AAA33694 & PrAS & Penicillium roqueforti & Aristolochene & (26) \\
\hline
\end{tabular}


Supplementary Table 4. Fungal TPSs used in this study not specified in Supplementary Table 3

\begin{tabular}{|c|c|}
\hline Ascomycota & JGI Trancript ID \\
\hline Acremonium chrysogenum ATCC 11550 & Acrchr1_8464 \\
\hline Alternaria alternata SRC1IrK2f v1.0 & Altal1_952578 \\
\hline Alternaria brassicicola & Altbr1_4174 \\
\hline \multirow{2}{*}{ Arthrobotrys oligospora ATCC 24927} & Artol1_6616 \\
\hline & Artol1_707 \\
\hline Ascocoryne sarcoides NRRL50072 & Ascsa1_6084 \\
\hline \multirow{2}{*}{ Aspergillus aculeatinus CBS 121060 v1.0 } & Aspacu1_414546 \\
\hline & Aspacu1_434153 \\
\hline \multirow{3}{*}{ Aspergillus bombycis NRRL 26010} & Aspbom1_115 \\
\hline & Aspbom1_5481 \\
\hline & Aspbom1_8488 \\
\hline \multirow{2}{*}{ Aspergillus brasiliensis v1.0 } & Aspbr1_199927 \\
\hline & Aspbr1_67363 \\
\hline \multirow{2}{*}{ Aspergillus carbonarius ITEM 5010 v3 } & Aspca3_399843 \\
\hline & Aspca3_517619 \\
\hline Aspergillus campestris IBT $28561 \mathrm{v} 1.0$ & Aspcam1_281577 \\
\hline Aspergillus candidus CBS 102.13 v1.0 & Aspcand1_97717 \\
\hline \multirow{2}{*}{ Aspergillus clavatus NRRL 1 from AspGD } & Aspcl1_3531 \\
\hline & Aspcl1_4382 \\
\hline Aspergillus costaricaensis CBS 115574 v1.0 & Aspcos1_212786 \\
\hline Aspergillus ellipticus CBS 707.79 v1.0 & Aspell1_449743 \\
\hline \multirow{2}{*}{ Aspergillus fijiensis CBS 313.89 v1.0 } & Aspfij1_393411 \\
\hline & Aspfij1_530370 \\
\hline Aspergillus flavus NRRL3357 & Aspfl1_31938 \\
\hline \multirow{2}{*}{ Aspergillus luchuensis CBS 106.47 v1.0 } & Aspfo1_40702 \\
\hline & Aspfo1_701451 \\
\hline Aspergillus heteromorphus CBS $117.55 \mathrm{v} 1.0$ & Asphet1_431411 \\
\hline Aspergillus ibericus CBS 121593 v1.0 & Aspibe1_454519 \\
\hline Aspergillus indologenus CBS 114.80 v1.0 & Aspind1_388859 \\
\hline \multirow{2}{*}{ Aspergillus kawachii IFO 4308} & Aspka1_1_18080 \\
\hline & Aspka1_1_21114 \\
\hline \multirow{2}{*}{ Aspergillus niger (lacticoffeatus) CBS 101883 v1.0 } & Asplac1_406332 \\
\hline & Asplac1_484152 \\
\hline Aspergillus neoniger CBS 115656 v1.0 & Aspneo1_451864 \\
\hline \multirow{2}{*}{ Aspergillus niger van Tieghem ATCC 13496 v1.0 } & Aspni_bvT_1_346145 \\
\hline & Aspni_bvT_1_349926 \\
\hline \multirow{2}{*}{ Aspergillus niger CBS 513.88} & Aspni_DSM_1_158750 \\
\hline & Aspni_DSM_1_163336 \\
\hline Aspergillus niger NRRL3 & Aspni_NRRL3_1_492 \\
\hline
\end{tabular}




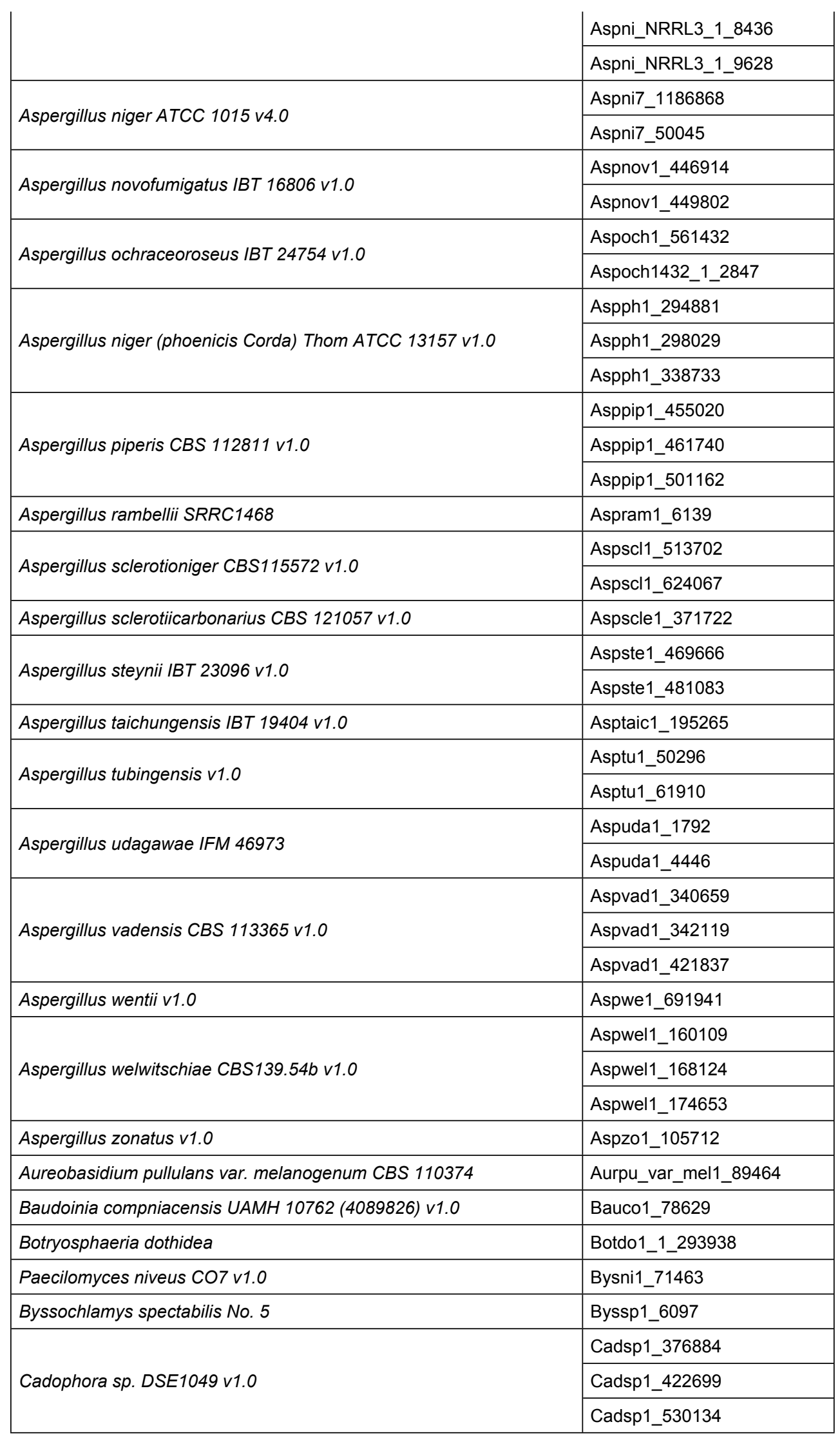




\begin{tabular}{|c|c|}
\hline & Cadsp1_589267 \\
\hline & Cadsp1_594154 \\
\hline Chaetomium globosum v1.0 & Chagl_1_13333 \\
\hline Cladosporium sphaerospermum UM 843 & Clasph1_4977 \\
\hline Cochliobolus carbonum 26-R-13 v1.0 & Cocca1_101564 \\
\hline Cochliobolus heterostrophus C4 v1.0 & CocheC4_1_33119 \\
\hline Cochliobolus lunatus m118 v2.0 & Coclu2_21271 \\
\hline & Cocmi1_35735 \\
\hline COCHIODOIUS myaneamuS ATCL 44500 VI.0 & Cocmi1_93455 \\
\hline Cochliobolus sativus ND90Pr v1.0 & Cocsa1_26197 \\
\hline Cochliobolus victoriae FI3 v1.0 & Cocvi1_101142 \\
\hline Colletotrichum graminicola M1.001 & Colgr1_7711 \\
\hline Colletotrichum orbiculare $104-T$ & Colorb1_137955 \\
\hline Coniosporium apollinis CBS 100218 & Conap1_98963 \\
\hline Corynespora cassiicola CCP v1.0 & Corca1_497534 \\
\hline Daldinia eschscholzii EC12 v1.0 & DalEC12_1_24917 \\
\hline Dothistroma septosporum NZE10 v1.0 & Dotse1_83036 \\
\hline Eucorium füituuroi IMI 58280 & Fusfu1_2062 \\
\hline 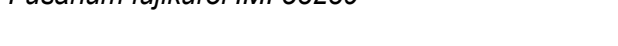 & Fusfu1_6471 \\
\hline Fusarium pseudograminearum CS3096 & Fusps1_11066 \\
\hline & Fusve2_19 \\
\hline Fusarium verticillioides 7600 v2 & Fusve2_20 \\
\hline & Fusve2_8588 \\
\hline Gaeumannomyces graminis var. tritici R3-111a-1 & Gaegr1_12383 \\
\hline & HypCl4A_1_10890 \\
\hline Hypoxylon sp. Cl-4A v1.0 & HypCl4A_1_216703 \\
\hline & HypCl4A_1_7273 \\
\hline Hypoxylon sp. EC38 v3.0 & HypEC38_3_428013 \\
\hline Hysterium pulicare & Hyspu1_1_111276 \\
\hline Hunnoctria on $\times 10$ & Ilysp1_1470883 \\
\hline 等 & Ilysp1_1672500 \\
\hline Magnaporthe grisea v1.0 & Maggr1_111583 \\
\hline Marssonina brunnea f. sp. multigermtubi MB_m1 & Marbr1_9792 \\
\hline Meliniomyces variabilis $F v 1.0$ & Melva1_456028 \\
\hline Microdochium bolleyi J235TASD1 v1.0 & Micbo1_186266 \\
\hline & Micca1_2230 \\
\hline Microsporum canis CBS 113480 & Micca1_5975 \\
\hline & Micca1_7780 \\
\hline Sh & Monha1_10324 \\
\hline 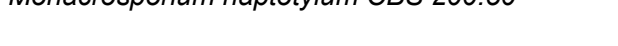 & Monha1_2682 \\
\hline Morchella importuna CCBAS932 v1.0 & Morco1_571997 \\
\hline
\end{tabular}




\begin{tabular}{|c|c|}
\hline Neosartorya fischeri NRRL 181 & Neofi1_1683 \\
\hline Oidiodendron maius Zn v1.0 & Oidma1_107908 \\
\hline Ophiostoma piceae UAMH 11346 & Ophpic1_6872 \\
\hline \multirow{2}{*}{ Paecilomyces variotii CBS144490 HYG1 v1.0 } & Paevar_HGY_1_328936 \\
\hline & Paevar1_455544 \\
\hline \multirow{2}{*}{ Paracoccidioides brasiliensis $\mathrm{PbO}$} & Parbr1_1519 \\
\hline & Parbra1_1841 \\
\hline Penicillium antarcticum IBT 31811 & Penant1_864 \\
\hline \multirow{2}{*}{ Penicillium chrysogenum v1.0 } & Pench1_6955 \\
\hline & Pench1_8552 \\
\hline Penicillium italicum PHI-1 & Penita1_363 \\
\hline Penicillium oxalicum 114-2 & Penox1_6322 \\
\hline Penicillium polonicum IBT 4502 & Penpol1_4238 \\
\hline Penicillium solitum IBT 29525 & Pensol1_10298 \\
\hline Penicillium thymicola DAOMC 180753 v1.0 & Penth1_187047 \\
\hline Penicillium vulpinum IBT 29486 & Penvul1_5047 \\
\hline Periconia macrospinosa DSE2036 v1.0 & Perma1_702268 \\
\hline \multirow{3}{*}{ Phialocephala scopiformis 5WS22E1 v1.0 } & Phisc1_723084 \\
\hline & Phisc1_757265 \\
\hline & Phisc1_779952 \\
\hline \multirow{2}{*}{ Pseudomassariella vexata CBS $129021 \mathrm{v} 1.0$} & Pseve2_344460 \\
\hline & Pseve2_356070 \\
\hline Pyrenophora teres f. teres & Pyrtt1_7556 \\
\hline \multirow{2}{*}{ Rhizoscyphus ericae UAMH 7357 v1.0 } & Rhier1_616373 \\
\hline & Rhier1_704773 \\
\hline Rhytidhysteron rufulum & Rhyru1_1_111615 \\
\hline Stagonospora sp. SRC1/sM3a v1.0 & Stasp1_276760 \\
\hline Talaromyces marneffei ATCC 18224 & Talma1_2_9641 \\
\hline Thielavia terrestris v2.0 & Thite2_2110120 \\
\hline Trichoderma arundinaceum IBT 40837 & Triaru1_10020 \\
\hline \multirow{2}{*}{ Trichoderma arundinaceum IBT 40837} & Triaru1_10784 \\
\hline & Triaru1_1442 \\
\hline Trichoderma asperellum CBS 433.97 v1.0 & Trias1_151771 \\
\hline Trichoderma asperellum TR356 v1.0 & Triasp1_382750 \\
\hline Trichoderma atroviride v2.0 & Triat2_86577 \\
\hline \multirow{2}{*}{ Trichoderma atrobrunneum ITEM 908} & Triatrob1_88627 \\
\hline & Triatrob1_89096 \\
\hline \multirow{3}{*}{ Trichoderma brevicompactum IBT40841 } & Tribre1_116064 \\
\hline & Tribre1_116420 \\
\hline & Tribre1_118439 \\
\hline Trichoderma citrinoviride TUCIM 6016 v4.0 & Trici4_1108344 \\
\hline
\end{tabular}




\begin{tabular}{|c|c|}
\hline & Trici4_1161166 \\
\hline & Trici4_1182837 \\
\hline & Trici4_66315 \\
\hline Trichoderma gamsii T6085 & Trigam1_162 \\
\hline & Triha1_497584 \\
\hline Trichoderma harzianum CBS 226.95 v1.0 & Triha1_502236 \\
\hline & Triha1_556193 \\
\hline & Trihar1_48479 \\
\hline & Trihar1_691447 \\
\hline 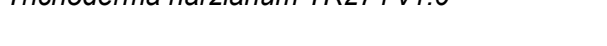 & Trihar1_703070 \\
\hline & Trihar1_828774 \\
\hline & Trilo3_1423477 \\
\hline Trichoderma longibrachiatum ATCC 18648 v3.0 & Trilo3_1442651 \\
\hline & Trilo3_1456781 \\
\hline & Tripar1_27 \\
\hline & Tripar1_4629 \\
\hline & Tripar1_6900 \\
\hline & Tripar1_7624 \\
\hline & Triple1_110780 \\
\hline 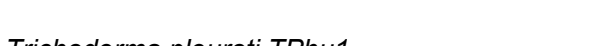 & Triple1_111606 \\
\hline 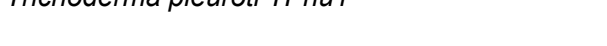 & Triple1_112591 \\
\hline & Triple1_116484 \\
\hline & Trire_Chr_111160 \\
\hline 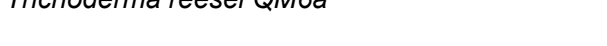 & Trire_Chr_117234 \\
\hline & Trire2_112028 \\
\hline Trichoderma reesei v2.0 & Trire2_122825 \\
\hline & Trire2_59597 \\
\hline & TrireRUTC30_1_10595 \\
\hline Trichoderma reesei RUT C-30 v1.0 & TrireRUTC30_1_12695 \\
\hline & TrireRUTC30_1_75235 \\
\hline Triahonhiton mbum CR 110000 & Triru1_3341 \\
\hline 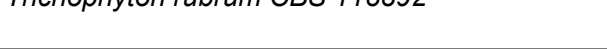 & Triru1_8324 \\
\hline & TriviGv29_8_2_141474 \\
\hline & TriviGv29_8_2_222187 \\
\hline Trichoderma virens Gv29-8 v2.0 & TriviGv29_8_2_225657 \\
\hline & TriviGv29_8_2_56195 \\
\hline & TriviGv29_8_2_59217 \\
\hline Venturia pirina & Venpi1_211641 \\
\hline Basidiomycota & JGI Trancript ID \\
\hline & Agabi_varbisH97_2_149463 \\
\hline & Agabi_varbisH97_2_183802 \\
\hline
\end{tabular}




\begin{tabular}{|c|c|}
\hline & Agabi_varbisH97_2_195544 \\
\hline & Agabi_varbisH97_2_208101 \\
\hline & Agabi_varbisH97_2_73543 \\
\hline & Agabi_varbur_1_109605 \\
\hline & Agabi_varbur_1_130532 \\
\hline & Agabi_varbur_1_46681 \\
\hline & Agabi_varbur_1_61902 \\
\hline & Agabi_varbur_1_76352 \\
\hline \multirow{11}{*}{ Amanita muscaria Koide v1.0 } & Amamu1_10014 \\
\hline & Amamu1_10025 \\
\hline & Amamu1_10032 \\
\hline & Amamu1_162077 \\
\hline & Amamu1_167471 \\
\hline & Amamu1_181219 \\
\hline & Amamu1_187046 \\
\hline & Amamu1_457766 \\
\hline & Amamu1_74562 \\
\hline & Amamu1_74856 \\
\hline & Amamu1_78657 \\
\hline \multirow{5}{*}{ Amanita thiersii Skay4041 v1.0 } & Amath1_2790 \\
\hline & Amath1_3814 \\
\hline & Amath1_55557 \\
\hline & Amath1_71495 \\
\hline & Amath1_77225 \\
\hline \multirow{17}{*}{ Armillaria cepistipes B5 } & Armcep1_11393 \\
\hline & Armcep1_14021 \\
\hline & Armcep1_14309 \\
\hline & Armcep1_14310 \\
\hline & Armcep1_14312 \\
\hline & Armcep1_14313 \\
\hline & Armcep1_15932 \\
\hline & Armcep1_15943 \\
\hline & Armcep1_15946 \\
\hline & Armcep1_15977 \\
\hline & Armcep1_17654 \\
\hline & Armcep1_1860 \\
\hline & Armcep1_20661 \\
\hline & Armcep1_20902 \\
\hline & Armcep1_6210 \\
\hline & Armcep1_6219 \\
\hline & Armcep1_6236 \\
\hline
\end{tabular}




\begin{tabular}{|c|c|}
\hline & Armcep1_6238 \\
\hline & Armcep1_6261 \\
\hline & Armcep1_6267 \\
\hline & Armcep1_6282 \\
\hline & Armcep1_8841 \\
\hline & Armcep1_8858 \\
\hline & Armcep1_9161 \\
\hline & Armcep1_9342 \\
\hline & Armga1_1000547 \\
\hline & Armga1_1022683 \\
\hline & Armga1_1046076 \\
\hline & Armga1_1057287 \\
\hline & Armga1_1069110 \\
\hline & Armga1_1079420 \\
\hline & Armga1_1099451 \\
\hline & Armga1_1099541 \\
\hline & Armga1_1099652 \\
\hline Armillaria gallica $21-2$ v1.0 & Armga1_1167519 \\
\hline & Armga1_1169433 \\
\hline & Armga1_302792 \\
\hline & Armga1_307601 \\
\hline & Armga1_946493 \\
\hline & Armga1_965633 \\
\hline & Armga1_970048 \\
\hline & Armga1_979797 \\
\hline & Armga1_979800 \\
\hline & Armga1_991859 \\
\hline & Armme1_1_12826 \\
\hline & Armme1_1_172 \\
\hline & Armme1_1_2843 \\
\hline & Armme1_1_3758 \\
\hline Armillaria mellea DSM 3731 & Armme1_1_4490 \\
\hline & Armme1_1_477 \\
\hline & Armme1_1_5648 \\
\hline & Armme1_1_5678 \\
\hline & Armme1_1_7007 \\
\hline & Armost1_1020351 \\
\hline & Armost1_1020352 \\
\hline Armillaria solidipes $28-4$ v1.0 & Armost1_1044432 \\
\hline & Armost1_806052 \\
\hline & Armost1_877051 \\
\hline
\end{tabular}




\begin{tabular}{|c|c|}
\hline & Armost1_888638 \\
\hline & Armost1_914197 \\
\hline & Armost1_925563 \\
\hline & Armost1_949886 \\
\hline & Armost1_949889 \\
\hline & Armost1_958287 \\
\hline & Armost1_959463 \\
\hline & Armost1_959487 \\
\hline & Armost1_980420 \\
\hline & Armost1_995389 \\
\hline & Armost1_996101 \\
\hline & Armost1_996102 \\
\hline & Armosto1_251811 \\
\hline & Armosto1_251832 \\
\hline & Armosto1_252116 \\
\hline & Armosto1_252241 \\
\hline & Armosto1_254059 \\
\hline & Armosto1_255619 \\
\hline & Armosto1_256008 \\
\hline A millaris coto 01010 & Armosto1_257779 \\
\hline 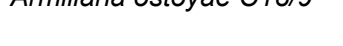 & Armosto1_257780 \\
\hline & Armosto1_263952 \\
\hline & Armosto1_263970 \\
\hline & Armosto1_263973 \\
\hline & Armosto1_263975 \\
\hline & Armosto1_263982 \\
\hline & Armosto1_263988 \\
\hline & Armosto1_272690 \\
\hline & Aurde3_1_1168918 \\
\hline & Aurde3_1_1233234 \\
\hline & Aurde3_1_1233260 \\
\hline Auricularia subglabra v2.0 & Aurde3_1_1241084 \\
\hline & Aurde3_1_1363163 \\
\hline & Aurde3_1_1415439 \\
\hline & Aurde3_1_63007 \\
\hline & Bjead1_1_105675 \\
\hline & Bjead1_1_156494 \\
\hline & Bjead1_1_166232 \\
\hline - Derkantuera auusta vi.u & Bjead1_1_337482 \\
\hline & Bjead1_1_53269 \\
\hline & Bjead1_1_65159 \\
\hline
\end{tabular}




\begin{tabular}{|c|c|}
\hline \multirow{6}{*}{ Botryobasidium botryosum v1.0 } & Botbo1_115416 \\
\hline & Botbo1_147726 \\
\hline & Botbo1_150564 \\
\hline & Botbo1_178061 \\
\hline & Botbo1_189792 \\
\hline & Botbo1_35207 \\
\hline \multirow{4}{*}{ Calocera cornea v1.0 } & Calco1_485261 \\
\hline & Calvi1_546336 \\
\hline & Calvi1_549380 \\
\hline & Calvi1_565634 \\
\hline \multirow{9}{*}{ Ceriporiopsis (Gelatoporia) subvermispora B } & Cersu1_108468 \\
\hline & Cersu1_110076 \\
\hline & Cersu1_119888 \\
\hline & Cersu1_120128 \\
\hline & Cersu1_128231 \\
\hline & Cersu1_138542 \\
\hline & Cersu1_173369 \\
\hline & Cersu1_85357 \\
\hline & Cersu1_85360 \\
\hline \multirow{16}{*}{ Coniophora olivacea MUCL 20566 v1.0 } & Conol1_187582 \\
\hline & Conol1_214788 \\
\hline & Conol1_623128 \\
\hline & Conol1_820881 \\
\hline & Conol1_828272 \\
\hline & Conol1_834476 \\
\hline & Conol1_837934 \\
\hline & Conol1_850378 \\
\hline & Conol1_916986 \\
\hline & Conol1_918725 \\
\hline & Conol1_918768 \\
\hline & Conol1_919993 \\
\hline & Conol1_923775 \\
\hline & Conol1_972285 \\
\hline & Conol1_980431 \\
\hline & Conol1_988075 \\
\hline \multirow{6}{*}{ Coniophora puteana v1.0 } & Conpu1_102303 \\
\hline & Conpu1_102358 \\
\hline & Conpu1_119051 \\
\hline & Conpu1_155276 \\
\hline & Conpu1_168744 \\
\hline & Conpu1_47835 \\
\hline
\end{tabular}




\begin{tabular}{|c|c|}
\hline & Conpu1_51079 \\
\hline & Conpu1_58147 \\
\hline & Conpu1_59039 \\
\hline & Conpu1_59132 \\
\hline & Conpu1_60589 \\
\hline & Conpu1_62857 \\
\hline & Conpu1_63141 \\
\hline & Conpu1_92329 \\
\hline & Copci1_20286 \\
\hline 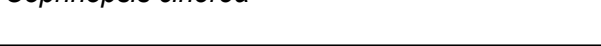 & Copci1_3117 \\
\hline & Copmar1_226928 \\
\hline & Copmar1_635391 \\
\hline 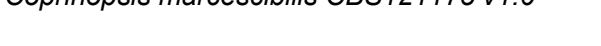 & Copmar1_660016 \\
\hline & Copmar1_663894 \\
\hline & Copmic2_1619241 \\
\hline & Copmic2_1626263 \\
\hline Coprinellus micaceus FP101781 v2.0 & Copmic2_1730196 \\
\hline & Copmic2_1806953 \\
\hline & Copmic2_514080 \\
\hline Cronartium quercuum f. sp. fusiforme G11 v1.0 & Croqu1_663498 \\
\hline & Crula1_116428 \\
\hline & Crula1_248883 \\
\hline & Crula1_410951 \\
\hline & Crula1_606715 \\
\hline Crunibulum laou CRC $16627 \times 10$ & Crula1_635172 \\
\hline 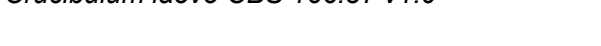 & Crula1_686927 \\
\hline & Crula1_704855 \\
\hline & Crula1_723748 \\
\hline & Crula1_737211 \\
\hline & Crula1_755525 \\
\hline & Cylto1_347558 \\
\hline & Cylto1_369898 \\
\hline & Cylto1_380850 \\
\hline & Cylto1_384854 \\
\hline Culindrohocidium torrondii ED15055 110 & Cylto1_401056 \\
\hline 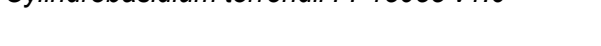 & Cylto1_405784 \\
\hline & Cylto1_436797 \\
\hline & Cylto1_442945 \\
\hline & Cylto1_453290 \\
\hline & Cylto1_453319 \\
\hline Dacryopinax primogenitus DJM 731 SSP1 v1.0 & Dacsp1_109748 \\
\hline
\end{tabular}




\begin{tabular}{|c|c|}
\hline & Dacsp1_34752 \\
\hline & Dacsp1_81273 \\
\hline & Daequ1_261869 \\
\hline & Daequ1_662999 \\
\hline & Daequ1_663158 \\
\hline Daedalea quercina v1.0 & Daequ1_678088 \\
\hline & Daequ1_737801 \\
\hline & Daequ1_769841 \\
\hline & Daequ1_811232 \\
\hline & Denbi1_650725 \\
\hline & Denbi1_655013 \\
\hline & Denbi1_659138 \\
\hline & Denbi1_659367\# \\
\hline & Denbi1_665533 \\
\hline & Denbi1_665578 \\
\hline & Denbi1_668482 \\
\hline & Denbi1_672591 \\
\hline & Denbi1_677017 \\
\hline & Denbi1_678887 \\
\hline & Denbi1_688396 \\
\hline & Denbi1_690040 \\
\hline & Denbi1_690806 \\
\hline & Denbi1_692335 \\
\hline & Denbi1_692909 \\
\hline 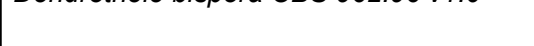 & Denbi1_694427 \\
\hline & Denbi1_694437 \\
\hline & Denbi1_701303 \\
\hline & Denbi1_704437 \\
\hline & Denbi1_816208\# \\
\hline & Denbi1_750593 \\
\hline & Denbi1_792840 \\
\hline & Denbi1_806729 \\
\hline & Denbi1_819488 \\
\hline & Denbi1_823222 \\
\hline & Denbi1_824683 \\
\hline & Denbi1_853901 \\
\hline & Denbi1_855582 \\
\hline & Denbi1_859389 \\
\hline & Denbi1_866930 \\
\hline nmitun naunlone ly & Dicsq1_138699 \\
\hline & Dicsq1_144692 \\
\hline
\end{tabular}




\begin{tabular}{|c|c|}
\hline & Dicsq1_146653 \\
\hline & Dicsq1_147860 \\
\hline & Dicsq1_159942 \\
\hline & Dicsq1_57946 \\
\hline & Dicsq1_58248 \\
\hline & Dicsq1_63388 \\
\hline & Dicsq1_80400 \\
\hline & Dicsq1_80593 \\
\hline & Dicsqu18370_1_442579 \\
\hline & Dicsqu18370_1_544240 \\
\hline & Dicsqu18370_1_652582 \\
\hline Dichomitus squalens OM18370.1 v1.0 & Dicsqu18370_1_662131 \\
\hline & Dicsqu18370_1_686806 \\
\hline & Dicsqu18370_1_744150 \\
\hline & Dicsqu18370_1_855629 \\
\hline & Dicsqu463_1_1009143 \\
\hline & Dicsqu463_1_399922 \\
\hline & Dicsqu463_1_849531 \\
\hline & Dicsqu463_1_851062 \\
\hline & Dicsqu463_1_862711 \\
\hline & Dicsqu463_1_909251 \\
\hline Dichomitus squalens CBS463.89 v1.0 & Dicsqu463_1_920193 \\
\hline & Dicsqu463_1_953290 \\
\hline & Dicsqu463_1_971284 \\
\hline & Dicsqu464_1_1013465 \\
\hline & Dicsqu464_1_690896 \\
\hline & Dicsqu464_1_961070 \\
\hline & Dicsqu464_1_971650 \\
\hline & Exigl1_605485 \\
\hline & Exigl1_611827 \\
\hline & Exigl1_620215 \\
\hline & Exigl1_665094 \\
\hline & Exigl1_673231 \\
\hline & Exigl1_673364 \\
\hline Exidia glandulosa v1.0 & Exigl1_678067 \\
\hline & Exigl1_678097 \\
\hline & Exigl1_681733 \\
\hline & Exigl1_682659 \\
\hline & Exigl1_688241 \\
\hline & Exigl1_713476 \\
\hline & Exigl1_743384 \\
\hline
\end{tabular}




\begin{tabular}{|c|c|}
\hline & Exigl1_750684 \\
\hline & Exigl1_767282 \\
\hline & Exigl1_769763 \\
\hline & Exigl1_769765 \\
\hline & Exigl1_770780 \\
\hline & Exigl1_776667 \\
\hline & Fibra1_4064 \\
\hline & Fibra1_4870 \\
\hline & Fibra1_5980 \\
\hline & Fibra1_6714 \\
\hline Fibroporia radiculosa TFFH 294 & Fibra1_679 \\
\hline & Fibra1_681 \\
\hline & Fibra1_7576 \\
\hline & Fibra1_7578 \\
\hline & Fibra1_8978 \\
\hline & Fibsp1_768221 \\
\hline & Fibsp1_820585 \\
\hline 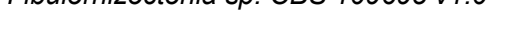 & Fibsp1_832739 \\
\hline & Fibsp1_943702 \\
\hline & Fishe1_34938 \\
\hline Fictulino honotion 10 & Fishe1_45577 \\
\hline 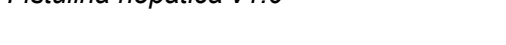 & Fishe1_46418 \\
\hline & Fishe1_66406 \\
\hline & Fomme1_105459 \\
\hline & Fomme1_109399 \\
\hline & Fomme1_112527 \\
\hline & Fomme1_170209 \\
\hline & Fomme1_17305 \\
\hline & Fomme1_27164 \\
\hline & Fomme1_80132 \\
\hline Fomitiporia mediterranea v1.0 & Fomme1_80285 \\
\hline & Fomme1_80525 \\
\hline & Fomme1_82160 \\
\hline & Fomme1_82892 \\
\hline & Fomme1_89879 \\
\hline & Fomme1_91887 \\
\hline & Fomme1_95474 \\
\hline & Fomme1_97142 \\
\hline & Fompi3_1017321 \\
\hline Fomitopsis pinicola FP-58527 SS1 v3.0 & Fompi3_1017322 \\
\hline & Fompi3_1023716 \\
\hline
\end{tabular}




\begin{tabular}{|c|c|}
\hline & Fompi3_1034271 \\
\hline & Fompi3_1048039 \\
\hline & Fompi3_1054994 \\
\hline & Fompi3_1118553 \\
\hline & Fompi3_1118777 \\
\hline & Fompi3_1120393 \\
\hline & Fompi3_1137037 \\
\hline & Fompi3_88323 \\
\hline & Galma1_104215\# \\
\hline & Galma1_1278553 \\
\hline & Galma1_1352450 \\
\hline & Galma1_137181 \\
\hline & Galma1_137592 \\
\hline & Galma1_141850 \\
\hline & Galma1_144010 \\
\hline & Galma1_222178 \\
\hline & Galma1_223839 \\
\hline & Galma1_225827 \\
\hline 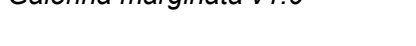 & Galma1_229350 \\
\hline & Galma1_245994 \\
\hline & Galma1_266943 \\
\hline & Galma1_62701 \\
\hline & Galma1_63702 \\
\hline & Galma1_63705 \\
\hline & Galma1_72483 \\
\hline & Galma1_72546 \\
\hline & Galma1_78619 \\
\hline & Galma1_78936 \\
\hline & Gansp1_106432 \\
\hline & Gansp1_119407 \\
\hline & Gansp1_126935 \\
\hline & Gansp1_144103 \\
\hline & Gansp1_151536 \\
\hline & Gansp1_164995 \\
\hline 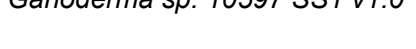 & Gansp1_167180 \\
\hline & Gansp1_41273 \\
\hline & Gansp1_57346 \\
\hline & Gansp1_57916 \\
\hline & Gansp1_58395 \\
\hline & Gansp1_59118 \\
\hline Gloeophyllum trabeum v1.0 & Glotr1_1_104020 \\
\hline
\end{tabular}




\begin{tabular}{|c|c|}
\hline & Glotr1_1_116368 \\
\hline & Glotr1_1_117462 \\
\hline & Glotr1_1_132121 \\
\hline & Glotr1_1_47776 \\
\hline & Glotr1_1_48421 \\
\hline & Glotr1_1_64303 \\
\hline & Glotr1_1_78603 \\
\hline & Glotr1_1_80048 \\
\hline & Glotr1_1_80521 \\
\hline & Gymlu1_152769 \\
\hline & Gymlu1_164762 \\
\hline & Gymlu1_179917 \\
\hline & Gymlu1_181444 \\
\hline & Gymlu1_239978 \\
\hline & Gymlu1_240888 \\
\hline 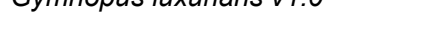 & Gymlu1_240889 \\
\hline & Gymlu1_240896 \\
\hline & Gymlu1_258218 \\
\hline & Gymlu1_70754 \\
\hline & Gymlu1_74399 \\
\hline & Gymlu1_775547 \\
\hline & Hebcy2_27568 \\
\hline & Hebcy2_32955 \\
\hline 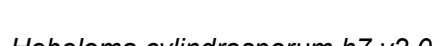 & Hebcy2_415352 \\
\hline 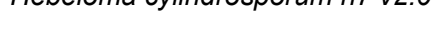 & Hebcy2_443163 \\
\hline & Hebcy2_7811 \\
\hline & Hebcy2_83676 \\
\hline & Helsul1_1632915 \\
\hline & Helsul1_1633994 \\
\hline & Helsul1_1719451 \\
\hline & Helsul1_1728450 \\
\hline 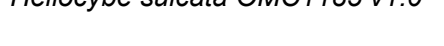 & Helsul1_1747561 \\
\hline & Helsul1_1811135 \\
\hline & Helsul1_291332 \\
\hline & Helsul1_470988 \\
\hline & Hetan2_148791 \\
\hline & Hetan2_167573 \\
\hline & Hetan2_34201 \\
\hline nelemonasianom anmosum va.0 & Hetan2_382802 \\
\hline & Hetan2_382866 \\
\hline & Hetan2_42859 \\
\hline
\end{tabular}




\begin{tabular}{|c|c|}
\hline & Hetan2_446121 \\
\hline & Hetan2_454193\# \\
\hline & Hetan2_458479 \\
\hline & Hetan2_48772 \\
\hline & Hetan2_51706 \\
\hline & Hydpi2_175482 \\
\hline & Hydpi2_31177 \\
\hline & Hydpi2_31185 \\
\hline & Hydpi2_85341 \\
\hline 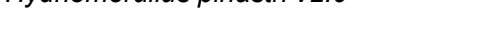 & Hydpi2_85943 \\
\hline & Hydpi2_90647 \\
\hline & Hydpi2_93582 \\
\hline & Hydpi2_95957 \\
\hline & Hypsu1_138395 \\
\hline & Hypsu1_138665\#* \\
\hline & Hypsu1_148594 \\
\hline Hypholoma sublateritium v1.0 & Hypsu1_148614 \\
\hline & Hypsu1_159625 \\
\hline & Hypsu1_202912 \\
\hline & Hypsu1_36696 \\
\hline & Jaaar1_125355 \\
\hline & Jaaar1_129201 \\
\hline & Jaaar1_162263 \\
\hline & Jaaar1_191537 \\
\hline lonnis sraillanon 10 & Jaaar1_206785 \\
\hline 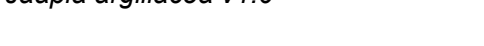 & Jaaar1_35496 \\
\hline & Jaaar1_453548 \\
\hline & Jaaar1_47267 \\
\hline & Jaaar1_488110 \\
\hline & Jaaar1_62205 \\
\hline & Lacam2_108968 \\
\hline & Lacam2_127112 \\
\hline & Lacam2_133376 \\
\hline Laccaria amethystina LaAM-08-1 v2.0 & Lacam2_310075 \\
\hline & Lacam2_619589 \\
\hline & Lacam2_682034 \\
\hline & Lacam2_99819 \\
\hline & Lacbi2_312850 \\
\hline I omonrin hisolory? & Lacbi2_393120 \\
\hline 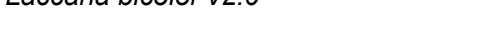 & Lacbi2_394925 \\
\hline & Lacbi2_443788 \\
\hline
\end{tabular}




\begin{tabular}{|c|c|}
\hline & Lacbi2_484390 \\
\hline \multirow{11}{*}{ Laetiporus sulphureus var. sulphureus v1.0 } & Laesu1_657387 \\
\hline & Laesu1_657801 \\
\hline & Laesu1_682308 \\
\hline & Laesu1_706476 \\
\hline & Laesu1_724793 \\
\hline & Laesu1_736396 \\
\hline & Laesu1_739130 \\
\hline & Laesu1_754875 \\
\hline & Laesu1_760870 \\
\hline & Laesu1_760873 \\
\hline & Laesu1_97318 \\
\hline \multirow{4}{*}{ Lentinula edodes B17 v1.1 } & Lened_B_1_1_15827 \\
\hline & Lened_B_1_1_3552 \\
\hline & Lened_B_1_1_6336 \\
\hline & Lened_B_1_1_884 \\
\hline \multirow{6}{*}{ Lentinula edodes W1-26 v1.0 } & Lentinedodes1_10218 \\
\hline & Lentinedodes1_12250 \\
\hline & Lentinedodes1_15846 \\
\hline & Lentinedodes1_4313 \\
\hline & Lentinedodes1_5399 \\
\hline & Lentinedodes1_6681 \\
\hline \multirow{3}{*}{ Leucoagaricus gongylophorus Ac12 } & Leugo1_1_50902 \\
\hline & Leugo1_1_51977 \\
\hline & Leugo1_1_52530 \\
\hline \multirow{11}{*}{ Peniophora sp. CONTA v1.0 } & Lopni1_247936 \\
\hline & Lopni1_557427 \\
\hline & Lopni1_579743 \\
\hline & Lopni1_600636 \\
\hline & Lopni1_604325 \\
\hline & Lopni1_612957 \\
\hline & Lopni1_617141 \\
\hline & Lopni1_644452 \\
\hline & Lopni1_684930 \\
\hline & Lopni1_717262 \\
\hline & Lopni1_718005 \\
\hline \multirow{2}{*}{ Moniliophthora perniciosa FA553 } & Monpe1_1_81866 \\
\hline & Monpe1_1_90530 \\
\hline \multirow{3}{*}{ Neolentinus lepideus v1.0 } & Neole1_1114335 \\
\hline & Neole1_1127639 \\
\hline & Neole1_1129682 \\
\hline
\end{tabular}




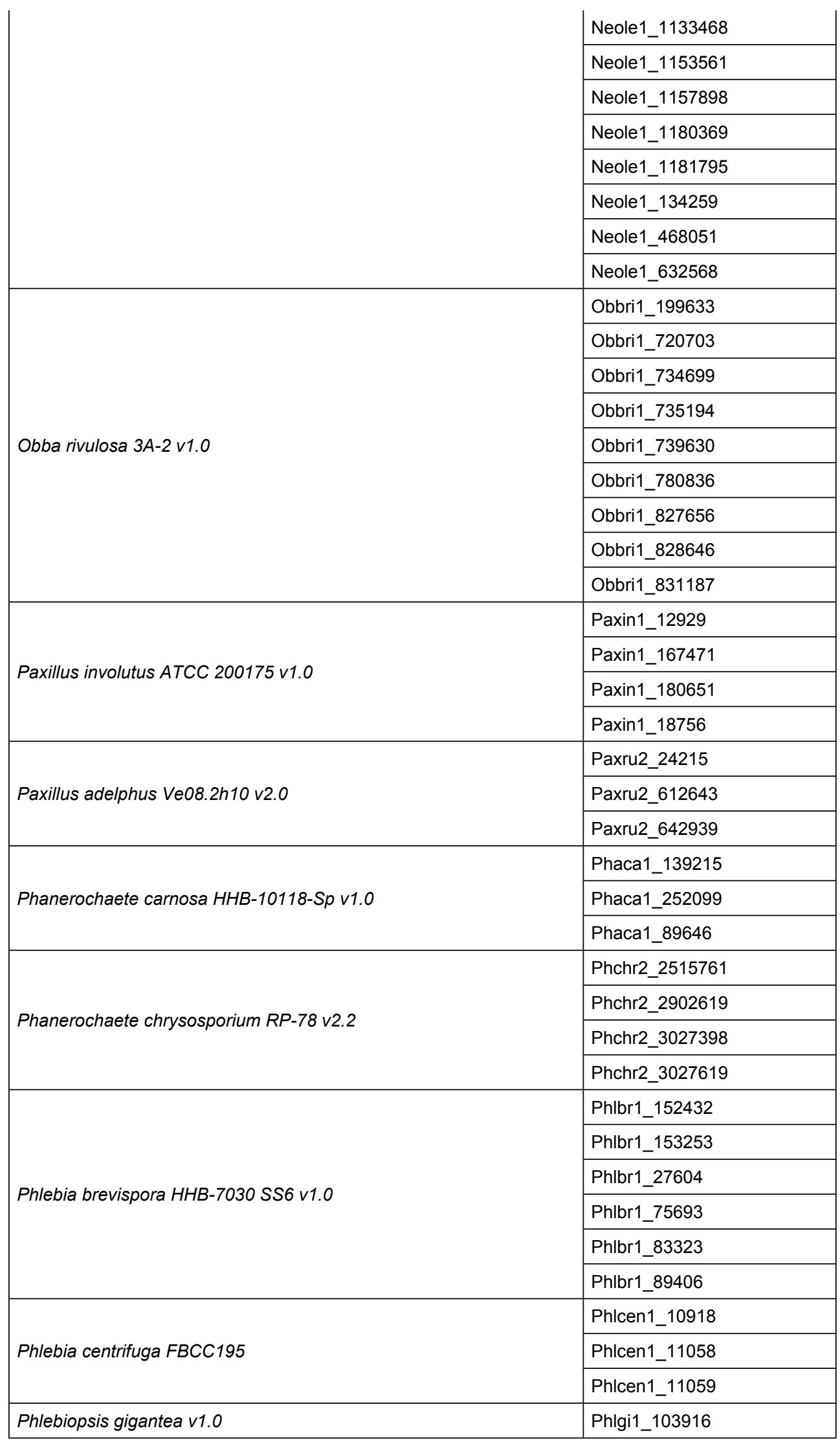




\begin{tabular}{|c|c|}
\hline & Phlgi1_114995 \\
\hline & Phlgi1_126910 \\
\hline & Phlgi1_157883 \\
\hline & Phlgi1_359236 \\
\hline & Phlgi1_367887 \\
\hline & Phlrad1_149880 \\
\hline & Phlrad1_158140 \\
\hline & Phlrad1_158151 \\
\hline Phlebia radiata Fr. (isolate 79, FBCC0043) & Phlrad1_158152 \\
\hline & Phlrad1_159909 \\
\hline & Phlrad1_160363 \\
\hline & Phlrad1_160364 \\
\hline & Pilcr1_14683 \\
\hline & Pilcr1_780025 \\
\hline & Pilcr1_810805 \\
\hline Piloderma croceum F 1598 v1.0 & Pilcr1_81177 \\
\hline & Pilcr1_825684\# \\
\hline & Pilcr1_828757 \\
\hline & Pilcr1_99075 \\
\hline & Pismi1_111823 \\
\hline & Pismi1_546683 \\
\hline & Pismi1_636226 \\
\hline ש. & Pismi1_642616 \\
\hline & Pismi1_685391 \\
\hline & Pismi1_88172 \\
\hline & Pisti1_138072 \\
\hline & Pisti1_170237 \\
\hline Pisolithus tinctorius Marx 270 v1.0 & Pisti1_27179 \\
\hline & Pisti1_29917 \\
\hline & Pisti1_471392 \\
\hline & PleosPC9_1_126403 \\
\hline & PleosPC9_1_39483 \\
\hline Pleurotus ostreatus PC9 v1.0 & PleosPC9_1_44504 \\
\hline & PleosPC9_1_49713 \\
\hline & PleosPC9_1_50646 \\
\hline & Plicr1_108846 \\
\hline Plicaturopsis crispa v1.0 & Plicr1_112400 \\
\hline & Plicr1_561009 \\
\hline & Plucer1_762199 \\
\hline Pluteus cervinus NL-1719 v1.0 & Plucer1_776166 \\
\hline & Plucer1_835830 \\
\hline
\end{tabular}




\begin{tabular}{|c|c|}
\hline & Plucer1_840592 \\
\hline & Plucer1_871394 \\
\hline & Polar1_208990 \\
\hline & Polar1_22511 \\
\hline & Polar1_22929 \\
\hline & Polar1_477864 \\
\hline Polyporus arcularius v1.0 & Polar1_487104 \\
\hline & Polar1_502877 \\
\hline & Polar1_547588 \\
\hline & Polar1_572468 \\
\hline & Polar1_574434 \\
\hline & Polbr1_1019111 \\
\hline & Polbr1_132650 \\
\hline & Polbr1_1353927 \\
\hline Datur ham & Polbr1_1355244 \\
\hline e & Polbr1_1451592 \\
\hline & Polbr1_48557 \\
\hline & Polbr1_50034 \\
\hline & Polbr1_622781 \\
\hline & Pospl1_105496 \\
\hline & Pospl1_127863 \\
\hline & Pospl1_130417 \\
\hline & Pospl1_44163 \\
\hline Postia placenta MAD 698-R v1.0 & Pospl1_46699 \\
\hline & Pospl1_60326 \\
\hline & Pospl1_87954 \\
\hline & Pospl1_89105 \\
\hline & Pospl1_97252 \\
\hline & PospIRSB12_1_1061514 \\
\hline Routin promenta & PospIRSB12_1_1145701 \\
\hline 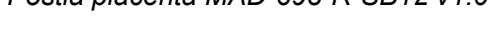 & PospIRSB12_1_1151376 \\
\hline & PospIRSB12_1_1155395 \\
\hline & Psicub1_1_31642 \\
\hline & Psicub1_1_32043 \\
\hline & Psicub1_1_33586 \\
\hline & Psicub1_1_35267 \\
\hline Psilocybe cubensis v1.0 & Psicub1_1_38644 \\
\hline & Psicub1_1_39291 \\
\hline & Psicub1_1_52546 \\
\hline & Psicub1_1_64449 \\
\hline & Psicub1_1_77598 \\
\hline
\end{tabular}




\begin{tabular}{|c|c|}
\hline & Psicub1_1_86860 \\
\hline & Psicub1_1_90383 \\
\hline & Psiser1_102197 \\
\hline & Psiser1_102363 \\
\hline & Psiser1_102395 \\
\hline & Psiser1_105633 \\
\hline & Psiser1_50890 \\
\hline & Psiser1_58778 \\
\hline Psilocybe serbica v1.0 & Psiser1_67725 \\
\hline & Psiser1_80182 \\
\hline & Psiser1_80775 \\
\hline & Psiser1_84069 \\
\hline & Psiser1_84646 \\
\hline & Psiser1_92040 \\
\hline & Psiser1_92157 \\
\hline & Ptegra1_308733 \\
\hline & Ptegra1_554786 \\
\hline Dtoulo arovilis CDC200 70410 & Ptegra1_556134 \\
\hline 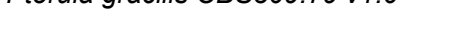 & Ptegra1_556592 \\
\hline & Ptegra1_573489 \\
\hline & Ptegra1_657455 \\
\hline & Punst1_108992 \\
\hline & Punst1_134858 \\
\hline & Punst1_146983 \\
\hline 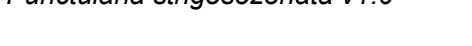 & Punst1_45111 \\
\hline & Punst1_62377 \\
\hline & Punst1_69113 \\
\hline & Pycci1_1170 \\
\hline & Pycci1_2035 \\
\hline & Pycci1_2731 \\
\hline Pycnoporus cinnabarinus BRFM 137 & Pycci1_5792 \\
\hline & Pycci1_6055 \\
\hline & Pycci1_6399 \\
\hline & Pycci1_6403 \\
\hline & Rhiso1_10420 \\
\hline & Rhiso1_1153 \\
\hline & Rhiso1_4580 \\
\hline & Rhiso1_4601 \\
\hline & Rhiso1_8981 \\
\hline & Rhiso1_9295 \\
\hline Rhizopogon vesiculosus Smith & Rhives1_11978 \\
\hline
\end{tabular}




\begin{tabular}{|c|c|}
\hline & Rhives1_134 \\
\hline & Rhives1_412 \\
\hline & Rhives1_414 \\
\hline & Rhives1_5856 \\
\hline & Rhives1_60 \\
\hline & Rhives1_7550 \\
\hline & Rhives1_7551 \\
\hline & Rhives1_8008 \\
\hline & Rhives1_8527 \\
\hline & Rhives1_8528 \\
\hline & Rhives1_9058 \\
\hline & Rhives1_9613 \\
\hline & Rhives1_9713 \\
\hline & Rhivi1_176640 \\
\hline & Rhivi1_339860 \\
\hline & Rhivi1_391988 \\
\hline & Rhivi1_537145 \\
\hline & Rhivi1_537178 \\
\hline & Rhivi1_574182 \\
\hline 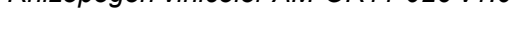 & Rhivi1_696738 \\
\hline & Rhivi1_699477 \\
\hline & Rhivi1_740933 \\
\hline & Rhivi1_767822 \\
\hline & Rhivi1_791528 \\
\hline & Rhivi1_849940 \\
\hline & Ricme1_573026 \\
\hline & Ricme1_601449 \\
\hline & Ricme1_625870 \\
\hline & Ricme1_627204 \\
\hline & Ricme1_636351 \\
\hline Peniophora sp. v1.0 & Ricme1_659570 \\
\hline & Ricme1_682875 \\
\hline & Ricme1_706833 \\
\hline & Ricme1_742269 \\
\hline & Ricme1_749414 \\
\hline & Ricme1_755282 \\
\hline & Ricmel1_601341 \\
\hline & Ricmel1_726626 \\
\hline Rickenella mellea v1.0 (SZMC22713) & Ricmel1_728269 \\
\hline & Ricmel1_732723 \\
\hline & Ricmel1_806373 \\
\hline
\end{tabular}




\begin{tabular}{|c|c|}
\hline & Ricmel1_842688 \\
\hline \multirow{3}{*}{ Schizophyllum commune H4-8 v3.0 } & Schco3_2543384 \\
\hline & Schco3_2672575 \\
\hline & Schco3_2749230 \\
\hline \multirow{15}{*}{ Schizopora paradoxa KUC8140 v1.0 } & Schpa1_385314 \\
\hline & Schpa1_600696 \\
\hline & Schpa1_600720 \\
\hline & Schpa1_626619 \\
\hline & Schpa1_825769 \\
\hline & Schpa1_828616 \\
\hline & Schpa1_828688 \\
\hline & Schpa1_890415 \\
\hline & Schpa1_893792 \\
\hline & Schpa1_894973 \\
\hline & Schpa1_910754 \\
\hline & Schpa1_938380 \\
\hline & Schpa1_940802 \\
\hline & Schpa1_940803 \\
\hline & Schpa1_944340 \\
\hline \multirow{5}{*}{ Scleroderma citrinum Foug A v1.0 } & Sclci1_100564 \\
\hline & Sclci1_1207496 \\
\hline & Sclci1_12722 \\
\hline & Sclci1_131609 \\
\hline & Sclci1_135004 \\
\hline Sebacina vermifera MAFF 305830 v1.0 & Sebve1_328047 \\
\hline \multirow{5}{*}{ Serpula himantioides (S.lacrymans var shastensis) MUCL38935 v1.0 } & Serla_varsha1_31195 \\
\hline & Serla_varsha1_35246 \\
\hline & Serla_varsha1_35279 \\
\hline & Serla_varsha1_40845 \\
\hline & Serla_varsha1_41137 \\
\hline \multirow{6}{*}{ Serpula lacrymans S7.3 v2.0 } & SerlaS7_3_2_108414 \\
\hline & SerlaS7_3_2_108585 \\
\hline & SerlaS7_3_2_165924 \\
\hline & SerlaS7_3_2_175395 \\
\hline & SerlaS7_3_2_90456 \\
\hline & SerlaS7_9_2_1175456 \\
\hline \multirow{5}{*}{ Sistotremastrum niveocremeum HHB9708 ss-1 1.0} & Sisni1_412423 \\
\hline & Sisni1_413173 \\
\hline & Sisni1_417871 \\
\hline & Sisni1_419098 \\
\hline & Sisni1_419116 \\
\hline
\end{tabular}




\begin{tabular}{|c|c|}
\hline & Sisni1_420465 \\
\hline & Sisni1_445702 \\
\hline & Sisni1_446571 \\
\hline & Sisni1_455980 \\
\hline & Sisni1_467088 \\
\hline & Sisni1_475990 \\
\hline & Sisni1_486756 \\
\hline & Sisni1_490732 \\
\hline & Sisni1_511672 \\
\hline & Sisni1_511758 \\
\hline & Sisni1_534754 \\
\hline & Sissu1_1009341 \\
\hline & Sissu1_1027304 \\
\hline & Sissu1_1035986 \\
\hline & Sissu1_1035993 \\
\hline & Sissu1_1052163 \\
\hline & Sissu1_1061555 \\
\hline & Sissu1_1062417 \\
\hline Sictotromectrum suncicum 11 & Sissu1_1062426 \\
\hline 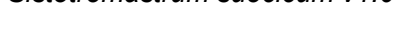 & Sissu1_1065835 \\
\hline & Sissu1_1067313 \\
\hline & Sissu1_1069570 \\
\hline & Sissu1_1132329 \\
\hline & Sissu1_138859 \\
\hline & Sissu1_221734 \\
\hline & Sissu1_992629 \\
\hline & Sissu1_993843 \\
\hline & Sphst1_150741 \\
\hline & Sphst1_182327 \\
\hline & Sphst1_182445 \\
\hline & Sphst1_185245 \\
\hline & Sphst1_193079 \\
\hline & Sphst1_239446 \\
\hline & Sphst1_256831 \\
\hline 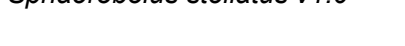 & Sphst1_256873 \\
\hline & Sphst1_258394 \\
\hline & Sphst1_263856 \\
\hline & Sphst1_265893 \\
\hline & Sphst1_267238 \\
\hline & Sphst1_267275 \\
\hline & Sphst1_268999 \\
\hline
\end{tabular}




\begin{tabular}{|c|c|}
\hline & Sphst1_47084\# \\
\hline & Sphst1_56545 \\
\hline & Sphst1_69328 \\
\hline & Sphst1_786515 \\
\hline & Stehi1_155829 \\
\hline Stereum hirsutum FP-91666 SS1 v1.0 & Stehi1_45773 \\
\hline & Stehi1_70654 \\
\hline & Suibr2_1892060 \\
\hline & Suibr2_711926 \\
\hline & Suibr2_745421 \\
\hline & Suibr2_777613 \\
\hline Suillus brevipes Sb2 v2.0 & Suibr2_787071 \\
\hline & Suibr2_845809 \\
\hline & Suibr2_849407 \\
\hline & Suibr2_855125 \\
\hline & Suibr2_914178 \\
\hline & Suilu4_23823 \\
\hline & Suilu4_2769573 \\
\hline Suillus luteus UH-Slu-Lm8-n1 v3.0 & Suilu4_2783843 \\
\hline & Suilu4_2841677 \\
\hline & Suilu4_2845464 \\
\hline & Trapub1_12698 \\
\hline & Trapub1_14506 \\
\hline & Trapub1_4727 \\
\hline Tramotos nubocons ERCO725 & Trapub1_4728 \\
\hline 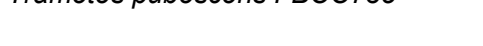 & Trapub1_6350 \\
\hline & Trapub1_6353 \\
\hline & Trapub1_7690 \\
\hline & Trapub1_9452 \\
\hline & Trave1_118446 \\
\hline & Trave1_119391 \\
\hline & Trave1_122474 \\
\hline & Trave1_125200 \\
\hline & Trave1_125951 \\
\hline 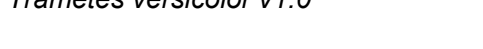 & Trave1_169361 \\
\hline & Trave1_21264 \\
\hline & Trave1_31247 \\
\hline & Trave1_35273 \\
\hline & Trave1_75848 \\
\hline 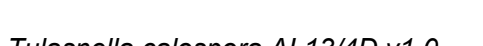 & Tulca1_214377 \\
\hline 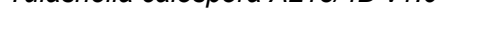 & Tulca1_214444 \\
\hline
\end{tabular}




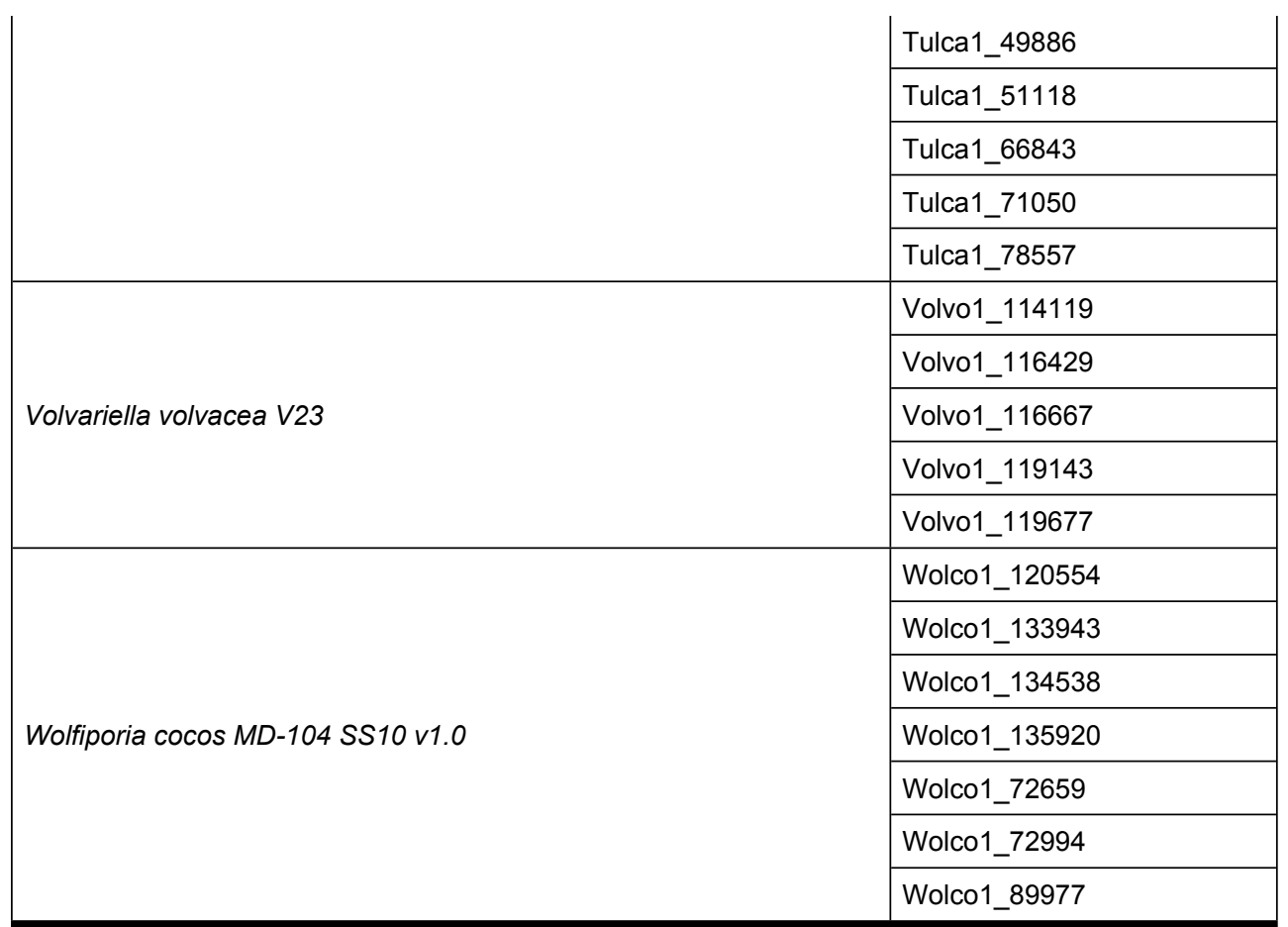

\# STSs with Protein IDs characterized in this study, ${ }^{*}$ sequence has been modified (see Figure 5)

Supplementary Table 5. NMR data of the isolated compound ( ${ }^{\mathrm{H}} \mathrm{H}$ : $600 \mathrm{MHz},{ }^{13 \mathrm{C}}$ : ${ }_{150} \mathrm{MHz}$; solvent: $\mathrm{CDCl}_{3}$ ).

\begin{tabular}{|l|l|l|l|l|}
\hline$\# \mathrm{C}$ & $\delta\left({ }^{13} \mathrm{C}\right)(\mathrm{ppm})$ & $\delta\left({ }^{1} \mathrm{H}\right)(\mathrm{ppm})$ & & $J$ \\
\hline 13 & 17.4 & $1.57(3 \mathrm{H}, \mathrm{br} . \mathrm{s})$ & $\mathrm{CH}_{3}$ & \\
\hline 12 & 20.5 & $1.06(3 \mathrm{H}, \mathrm{s})$ & $\mathrm{CH}_{3}$ & \\
\hline 5 & 25.5 & $\begin{array}{l}2.53(1 \mathrm{H}, \mathrm{m}) \alpha \\
2.72(1 \mathrm{H}, \mathrm{m}) \beta\end{array}$ & \} $\mathrm{CH}_{2}$ & \\
\hline 14 & 27.5 & $0.93(3 \mathrm{H}, \mathrm{s})$ & $\mathrm{CH}_{3}$ & \\
\hline 15 & 29.8 & $1.04(3 \mathrm{H}, \mathrm{s})$ & $\mathrm{CH}_{3}$ & \\
\hline 8 & 34.2 & $\begin{array}{l}1.65(1 \mathrm{H}, \mathrm{m}) \alpha \\
1.85(1 \mathrm{H}, \mathrm{dd}) \beta\end{array}$ & \} $\mathrm{CH}_{2}$ & $\mathrm{~J}=7,14 \mathrm{~Hz}$ \\
\hline 4 & 36.9 & $\begin{array}{l}1.74(1 \mathrm{H}, \mathrm{m}) \beta \\
1.81(1 \mathrm{H}, \mathrm{ddd}) \alpha\end{array}$ & \} $\mathrm{CH}_{2}$ & $J=3,9,11 \mathrm{~Hz}$ \\
\hline 11 & 39.2 & --- & $\mathrm{C}$ & \\
\hline 9 & 40.6 & $2.34(1 \mathrm{H}, \mathrm{qt}) \beta$ & $\mathrm{CH} \beta$ & $J=7,11 \mathrm{~Hz}$ \\
\hline 1 & 41.1 & $\begin{array}{l}1.29(1 \mathrm{H}, \mathrm{dd}) \alpha \\
1.35(1 \mathrm{H}, \mathrm{ddd}) \beta\end{array}$ & \} $\mathrm{CH}_{2}$ & $\mathrm{~J}=11,12 \mathrm{~Hz}$ \\
\hline 3 & 45.7 & -- & $\mathrm{C}$ & \\
\hline 2 & 47.1 & $2.16(1 \mathrm{H}, \mathrm{ddd}) \beta$ & $\mathrm{CH}_{12} \mathrm{~Hz}$ \\
\hline 10 & 48.6 & $\begin{array}{l}0.96(1 \mathrm{H}, \mathrm{m}) \alpha \\
1.53(1 \mathrm{H}, \mathrm{ddd}) \beta\end{array}$ & \} $\mathrm{CH}_{2}$ & $\mathrm{~J}=2,7,12 \mathrm{~Hz}$ \\
\hline 7 & 123.1 & -- & $\mathrm{C}$ & \\
\hline 6 & 141.8 & $---11 \mathrm{~Hz}$ \\
\hline
\end{tabular}




\section{Supplementary Table 6. Information of STSs in mid-group 5.}

\begin{tabular}{|c|c|c|c|}
\hline No. & Description & Species & Phyla \\
\hline 1 & Asppip1_461740 & Aspergillus piperis & Ascomycota \\
\hline 2 & Aspram1_6139 & Aspergillus rambellii & Ascomycota \\
\hline 3 & Aspfij1_530370 & Aspergillus fijiensis & Ascomycota \\
\hline 4 & Micca1_7780 & Microsporum canis & Ascomycota \\
\hline 5 & Aspind1_388859 & Aspergillus indologenus & Ascomycota \\
\hline 6 & Aspuda1_1792 & Aspergillus udagawae & Ascomycota \\
\hline 7 & Asplac1_406332 & Aspergillus niger & Ascomycota \\
\hline 8 & Triru1_3341 & Trichophyton rubrum & Ascomycota \\
\hline 9 & Aspvad1 421837 & Aspergillus vadensis & Ascomycota \\
\hline 10 & Aspni_bvT_1_349926 & Aspergillus niger & Ascomycota \\
\hline 11 & Aspwel1_168124 & Aspergillus welwitschiae & Ascomycota \\
\hline 12 & Penox1_6322 & Penicillium oxalicum & Ascomycota \\
\hline 13 & Aspni_NRRL3_1_9628 & Aspergillus niger & Ascomycota \\
\hline 14 & Aspacu1_414546 & Aspergillus aculeatinus & Ascomycota \\
\hline 15 & Aspbom1_8488 & Aspergillus bombycis & Ascomycota \\
\hline 16 & Aspnov1_449802 & Aspergillus novofumigatus & Ascomycota \\
\hline 17 & Aspbom1_115 & Aspergillus bombycis & Ascomycota \\
\hline 18 & Aspoch1_561432 & Aspergillus ochraceoroseus & Ascomycota \\
\hline 19 & Aspca3_399843 & Aspergillus carbonarius & Ascomycota \\
\hline 20 & Aspoch1432_1_2847 & Aspergillus ochraceoroseus & Ascomycota \\
\hline 21 & Aspph1_294881 & Aspergillus niger & Ascomycota \\
\hline
\end{tabular}

\section{REFERENCES}

1. Orban, A., Fraatz, M. A., and Rühl, M. (2019) Aroma Profile Analyses of Filamentous Fungi Cultivated on Solid Substrates, Adv. Biochem. Engin./Biotechnol. Epub 2019. DOI: 10.1007/10_2019_87.

2. Hanssen, H.-P., Sprecher, E., and Abraham, W.-R. (1986) 6-Protoilludene, the major volatile metabolite from ceratocystis piceae liquid cultures, Phytochem. 25, 1979-1980.

3. Nozoe, S., Kobayashi, H., Urano, S., and Furukawa, J. (1977) Isolation of $\Delta 6$-protoilludene and the related alcohols, Tetrahedron Letters 18, 1381-1384.

4. Gupta, D. K., Ruhl, M., Mishra, B., Kleofas, V., Hofrichter, M., Herzog, R., Pecyna, M. J., Sharma, R., Kellner, H., Hennicke, F., and Thines, M. (2018) The genome sequence of the commercially cultivated mushroom Agrocybe aegerita reveals a conserved repertoire of fruiting-related genes and a versatile suite of biopolymer-degrading enzymes, BMC Genomics 19, 48.

5. Grigoriev, I. V., Nikitin, R., Haridas, S., Kuo, A., Ohm, R., Otillar, R., Riley, R., Salamov, A., Zhao, X., Korzeniewski, F., Smirnova, T., Nordberg, H., Dubchak, I., and Shabalov, I. (2014) MycoCosm portal: gearing up for 1000 fungal genomes, Nucleic Acids Res. 42, D699-704.

6. Engels, B., Heinig, U., Grothe, T., Stadler, M., and Jennewein, S. (2011) Cloning and Characterization of an Armillaria gallica cDNA Encoding Protoilludene Synthase, Which Catalyzes the First Committed Step in the Synthesis of Antimicrobial Melleolides, J. Biochem. 286, 6871-6878.

7. Nagamine, S., Liu, C., Nishishita, J., Kozaki, T., Sogahata, K., Sato, Y., Minami, A., Ozaki, T., Schmidt-Dannert, C., Maruyama, J.-i., and Oikawa, H. (2019) Ascomycota Aspergillus oryzae is an efficient expression host for production of Basidiomycota terpenes using genomic DNA sequences, Appl. Environ. Microbiol. Epub 2019. DOI: 10.1128/AEM.oo409-19.

8. Mischko, W., Hirte, M., Fuchs, M., Mehlmer, N., and Brück, T. B. (2018) Identification of sesquiterpene synthases from the Basidiomycota Coniophora puteana for the efficient and highly selective $\beta$-copaene and cubebol production in $E$. coli, Microb. Cell Fact. 17, 164. 
9. Agger, S., Lopez-Gallego, F., and Schmidt-Dannert, C. (2009) Diversity of sesquiterpene synthases in the basidiomycete Coprinus cinereus, Mol. Microbiol. 72, 1181-1195.

10. Wawrzyn, G. T., Quin, M. B., Choudhary, S., Lopez-Gallego, F., and Schmidt-Dannert, C. (2012) Draft genome of Omphalotus olearius provides a predictive framework for sesquiterpenoid natural product biosynthesis in Basidiomycota, Chem. Biol. 19, 772-783.

11. Yap, H.-Y. Y., Muria-Gonzalez, M. J., Kong, B.-H., Stubbs, K. A., Tan, C.-S., Ng, S.-T., Tan, N.-H., Solomon, P. S., Fung, S.Y., and Chooi, Y.-H. (2017) Heterologous expression of cytotoxic sesquiterpenoids from the medicinal mushroom Lignosus rhinocerotis in yeast, Microb. Cell Fact. 16, 103.

12. Ichinose, H., and Kitaoka, T. (2018) Insight into metabolic diversity of the brown-rot basidiomycete Postia placenta responsible for sesquiterpene biosynthesis: semi-comprehensive screening of cytochrome $\mathrm{P} 450$ monooxygenase involved in protoilludene metabolism, Microb. Biotechnol. 11, 952-965.

13. Quin, M. B., Flynn, C. M., Wawrzyn, G. T., Choudhary, S., and Schmidt-Dannert, C. (2013) Mushroom hunting by using bioinformatics. application of a predictive framework facilitates the selective identification of sesquiterpene synthases in basidiomycota, Chembiochem 14, 2480-2491.

14. Burkhardt, I., Kreuzenbeck, N. B., Beemelmanns, C., and Dickschat, J. S. (2019) Mechanistic characterization of three sesquiterpene synthases from the termite-associated fungus Termitomyces, Org Biomol Chem 17, 3348-3355.

15. Yee, D. A., Kakule, T. B., Cheng, W., Chen, M., Chong, C. T. Y., Hai, Y., Hang, L. F., Hung, Y.-S., Liu, N., Ohasi, M., Okorafor, I. C., Song, Y., Tang, A., Zhang, Z., and Tang, Y. (2020) Genome Mining of Alkaloidal Terpenoids from a Hybrid Terpene and Nonribosomal Peptide Biosynthetic Pathway, J. Am. Chem. Soc. 142, 710-714.

16. Cane, D. E., and Kang, I. (2000) Aristolochene synthase. purification, molecular cloning, high-level expression in Escherichia coli, and characterization of the Aspergillus terreus cyclase, Arch. Biochem. Biophys. 376, 354-364.

17. Moraga, J., Dalmais, B., Izquierdo-Bueno, I., Aleu, J., Hanson, J. R., Hernández-Galán, R., Viaud, M., and Collado, I. G. (2016) Genetic and Molecular Basis of Botrydial Biosynthesis: Connecting Cytochrome P450-Encoding Genes to Biosynthetic Intermediates, ACS Chem. Biol. 11, 2838-2846.

18. Takino, J., Kozaki, T., Sato, Y., Liu, C., Ozaki, T., Minami, A., and Oikawa, H. (2018) Unveiling Biosynthesis of the Phytohormone Abscisic Acid in Fungi: Unprecedented Mechanism of Core Scaffold Formation Catalyzed by an Unusual Sesquiterpene Synthase, J. Am. Chem. Soc. 140, 12392-12395.

19. Wu, W., Tran, W., Taatjes, C. A., Alonso-Gutierrez, J., Lee, T. S., and Gladden, J. M. (2016) Rapid Discovery and Functional Characterization of Terpene Synthases from Four Endophytic Xylariaceae, PloS one 11, e0146983-19.

2o. de Sena Filho, Jose Guedes, Quin, M. B., Spakowicz, D. J., Shaw, J. J., Kucera, K., Dunican, B., Strobel, S. A., and SchmidtDannert, C. (2016) Genome of Diaporthe sp. provides insights into the potential inter-phylum transfer of a fungal sesquiterpenoid biosynthetic pathway, Fungal Biol. 120, 1050-1063.

21. Burkhardt, I., Siemon, T., Henrot, M., Studt, L., Rosler, S., Tudzynski, B., Christmann, M., and Dickschat, J. S. (2016) Mechanistic Characterisation of Two Sesquiterpene Cyclases from the Plant Pathogenic Fungus Fusarium fujikuroi, Angew. Chem. Int. Ed. Engl. 55, 8748-8751.

22. Brock, N. L., Huss, K., Tudzynski, B., and Dickschat, J. S. (2013) Genetic dissection of sesquiterpene biosynthesis by Fusarium fujikuroi, Chembiochem 14, 311-315.

23. McCormick, S. P., Alexander, N. J., and Harris, L. J. (2010) CLM1 of Fusarium graminearum encodes a longiborneol synthase required for culmorin production, Appl. Environ. Microbiol. 76, 136-141.

24. Rynkiewicz, M. J., Cane, D. E., and Christianson, D. W. (2001) Structure of trichodiene synthase from Fusarium sporotrichioides provides mechanistic inferences on the terpene cyclization cascade, PNAS 98, 13543-13548.

25. Shaw, J. J., Berbasova, T., Sasaki, T., Jefferson-George, K., Spakowicz, D. J., Dunican, B. F., Portero, C. E., Narvaez-Trujillo, A., and Strobel, S. A. (2015) Identification of a fungal 1,8-cineole synthase from Hypoxylon sp. with specificity determinants in common with the plant synthases, J. Biol. Chem. 290, 8511-8526.

26. Hohn, T. M., and Plattner, R. D. (1989) Purification and characterization of the sesquiterpene cyclase aristolochene synthase from Penicillium roqueforti, Arch. Biochem. Biophys. 272, 137-143. 